\title{
Semiclassical theory of weak antilocalization and spin relaxation in ballistic quantum dots
}

\author{
Oleg Zaitsev, ${ }^{1, *}$ Diego Frustaglia, ${ }^{2}$ and Klaus Richter ${ }^{1}$ \\ ${ }^{1}$ Institut für Theoretische Physik, Universität Regensburg, D-93040 Regensburg, Germany \\ ${ }^{2}$ NEST-INFM and Scuola Normale Superiore, 56126 Pisa, Italy
}

(Received 7 June 2005; published 25 October 2005)

\begin{abstract}
We develop a semiclassical theory for spin-dependent quantum transport in ballistic quantum dots. The theory is based on the semiclassical Landauer formula, that we generalize to include spin-orbit and Zeeman interaction. Within this approach, the orbital degrees of freedom are treated semiclassically, while the spin dynamics is computed quantum mechanically. Employing this method, we calculate the quantum correction to the conductance in quantum dots with Rashba and Dresselhaus spin-orbit interaction. We find a strong sensitivity of the quantum correction to the underlying classical dynamics of the system. In particular, a suppression of weak antilocalization in integrable systems is observed. These results are attributed to the qualitatively different types of spin relaxation in integrable and chaotic quantum cavities.
\end{abstract}

DOI: 10.1103/PhysRevB.72.155325

PACS number(s): 73.23.- b, 03.65.Sq, 71.70.Ej

\section{INTRODUCTION}

Guided by the vision to incorporate spin physics into the far-advanced semiconductor (hetero)structure technology, semiconductor-based spin electronics (see, e.g., Ref. 1) has developed into a prominent branch of present spintronics research. In this context spin-orbit (SO) interactions have recently received considerable attention since they give rise to rich spin dynamics and a variety of spin phenomena in nonmagnetic semiconductors. Though SO couplings have been a subject of continuous research throughout the last decades, ${ }^{2-8}$ there is presently a revival in investigating SO effects owing to their role in spin transistors, ${ }^{9,10}$ spin interferometers, ${ }^{11,12}$ spin filters, ${ }^{13,14}$ and spin pumps, ${ }^{15,16}$ to name only a few examples. Furthermore, most recently the intrinsic spin Hall effect ${ }^{17,18}$ in a SO-coupled system has caused an intense and controversial discussion in the literature. Finally, in spin-based quantum computation SOinduced spin relaxation effects may play a role. ${ }^{19}$

The interplay between spin dynamics and confinement effects is particularly intriguing in quantum transport through low-dimensional devices at low temperatures where quantum coherence effects additionally arise. There exist two prominent experimental probes for SO effects in quantum transport, (i) characteristic beating patterns in Shubnikov-de Haas oscillations in two-dimensional electron gases with tunable SO coupling, controlled via a back-gate voltage, ${ }^{20-23}$ and (ii) weak antilocalization ${ }^{24-27}$ (WA), an enhancement of the magnetoconductance at zero magnetic field owing to spindependent quantum interference effects. Since systems without SO coupling exhibit weak localization (WL), i.e., a reduction in the magnetoconductance, the appearance of WA allows conclusions to be drawn on the SO strength. While WA is fairly well understood for disordered bulk systems ${ }^{28-30}$ in recent experiments using ballistic bismuth ${ }^{31}$ and $\mathrm{GaAs}^{32}$ cavities, WA has been employed to study SOinduced spin dynamics and spin relaxation phenomena in confined systems. These measurements are focussed on the interesting interrelation between quantum confined orbital motion and spin evolution and relaxation in clean ballistic quantum dots. In these systems, the elastic mean free path is exceeding their size, and impurity scattering is replaced by reflections off the system boundaries.

Corresponding efforts in treating SO effects on spectra, ${ }^{33-36}$ spin relaxation, ${ }^{37-39}$ and the interplay between SO and Zeeman coupling ${ }^{40,41}$ in quantum dots have also been made on the theoretical side. SO-induced WA in ballistic quantum dots has been studied using random-matrix theory ${ }^{42,43}$ (RMT) and semiclassical approaches. ${ }^{44,45}$ While RMT approaches are restricted to quantum dots with corresponding chaotic classical dynamics, the semiclassical transport theory comprises a much broader class of systems, including integrable confinement geometries. Related semiclassical techniques have also been applied to spin transmission ${ }^{46}$ and spin relaxation ${ }^{47}$ in quantum dots.

The purpose of the present paper is a detailed exposition and extension of the semiclassical methods of Refs. 44 and 45. The theory to be discussed here unifies two subject areas, the semiclassical description of $\mathrm{WL}^{48-50}$ and the semiclassical treatment of SO interaction. ${ }^{51-57}$ Compared to the earlier works ${ }^{44,45,47}$ on the subject, here we give special attention to the differences in spin relaxation along open and closed trajectories, analyze the interplay between Rashba and Dresselhaus interaction, and report on the full quantum calculations of spin-dependent transmission and reflection.

This paper is organized as follows: In the introductory Sec. II, using path integrals with spin coherent states we deduce a spin-dependent semiclassical propagator and the corresponding Green function. Our main analytical results are presented in Sec. III. There, on the basis of Green functions a semiclassical approximation to the Landauer formula with spin is derived. The semiclassical Landauer formula is then applied to chaotic quantum dots, whereby the quantum corrections to transmission and reflection are calculated. In Sec. IV we discuss how WA is related to the spin evolution. We define measures for spin relaxation and consider, as an example, the spin relaxation in diffusive systems. In the following two sections the general theory is applied to chaotic and integrable quantum dots with Rashba and Dresselhaus SO interaction. In Sec. V a detailed numerical study of the spin relaxation is followed by an analysis of the limit of slow spin dynamics (i.e., extremely weak SO coupling). Addition- 
ally, we examine a gauge transformation of the spin-orbit Hamiltonian that can be carried out in this limit. The dependence of the quantum corrections to transmission and reflection on the SO-coupling strength and magnetic field (Aharonov-Bohm and Zeeman contributions) is presented in Sec. VI. There some of the semiclassical numerical results are compared with full-scale numerical quantum calculations.

\section{SPIN-ORBIT INTERACTION IN A SEMICLASSICAL THEORY}

In this preparatory section we construct a spin-dependent semiclassical propagator ${ }^{81}$ and related Green function. It fully describes the system at a given level of approximation and, thus, can serve as a starting point for our derivation of a semiclassical Landauer formula for systems with SO and Zeeman interaction (Sec. III).

In the spinless case, the semiclassical propagator is conventionally obtained from the path-integral representation of the exact propagator. ${ }^{58}$ After the stationary-phase evaluation, which is valid in the semiclassical limit, the classical trajectories are selected from all the paths in the integral. In order to include spin into the path integral, a continuous basis of spin states is required. The spin coherent states represent such a basis. ${ }^{59,60}$

Following Ref. 60, we define a coherent state of spin $s$ $=\frac{1}{2}, 1, \ldots$ by

$$
|\zeta\rangle=\left(1+|\zeta|^{2}\right)^{-s} \exp \left(\zeta \hat{s}_{+}\right)|\sigma=-s\rangle,
$$

where $\zeta$ is a complex number that labels the state, $\hat{s}_{+}=\hat{s}_{x}$ $+i \hat{s}_{y}$ is the spin operator, and $|\sigma\rangle$ are the eigenstates of $\hat{s}_{z}$ with eigenvalues $\sigma=-s, \ldots, s$. To each $\zeta$ corresponds a threedimensional unit vector $\mathbf{n}(\zeta)=\langle\zeta|\hat{\mathbf{s}}| \zeta\rangle / s$ that denotes the spin direction. It is easy to show that $\zeta$ is a stereographic projection from the unit sphere centered at the origin onto the plane $z=0$. The projection is given by $\left(n_{x}, n_{y}, n_{z}\right) \mapsto(\operatorname{Re} \zeta$, $-\operatorname{Im} \zeta, 0)$, where, in particular, the south pole is mapped to $\zeta=0$. In general, coherent states have the minimal uncertainty of $\hat{\mathbf{s}}$ among all spin states and are characterized by three real parameters: the direction $\mathbf{n}$ and an overall phase. (Hence, any state of spin $1 / 2$ is coherent.) In the current definition, the phase is assigned to each $\mathbf{n}$ by Eq. (1), but other phase assignments are possible. Note that the phase of the state $|\zeta=\infty\rangle \propto|\sigma=s\rangle$ with $\mathbf{n}=(0,0,1)$ is not well defined. However, this manifestation of the fundamental problem of phase assignment ${ }^{61}$ does not pose a difficulty in our case, since the final results will be transformed to the $|\sigma\rangle$ representation using the projection operators $|\zeta\rangle\langle\zeta|$. The states (1) are normalized to unity, but, obviously, not mutually orthogonal (no more than $2 s+1$ states of spin $s$ can be mutually orthogonal). Nevertheless, having the property of resolution of unity,

$$
\int|\zeta\rangle\langle\zeta| d \mu(\zeta)=\hat{1}, \quad d \mu(\zeta)=\frac{2 s+1}{\pi\left(1+|\zeta|^{2}\right)^{2}} d^{2} \zeta,
$$

they form an (overcomplete) basis and enable a path-integral construction.
Let us consider a rather general case of a system with Hamiltonian linear in the spin operator $\hat{\mathbf{s}}$,

$$
\hat{H}=\hat{H}^{0}(\hat{\mathbf{q}}, \hat{\mathbf{p}})+\hbar \hat{\mathbf{s}} \cdot \hat{\mathbf{C}}(\hat{\mathbf{q}}, \hat{\mathbf{p}}) \text {. }
$$

Here $\hat{\mathbf{q}}$ and $\hat{\mathbf{p}}$ are the $d$-dimensional coordinate and momentum operators, respectively, $\hat{H}^{0}(\hat{\mathbf{q}}, \hat{\mathbf{p}})$ is the spin-independent Hamiltonian, and $\hbar \hat{\mathbf{s}} \cdot \hat{\mathbf{C}}(\hat{\mathbf{q}}, \hat{\mathbf{p}})$ describes the SO interaction and the Zeeman interaction with an external (generally inhomogeneous) magnetic field. Utilizing the idea of Refs. 54 and 56, we express the propagator in the combined coordinate and spin-coherent-state representation in terms of the path integral,

$$
\begin{aligned}
U\left(\mathbf{q}_{2}, \zeta_{2}, \mathbf{q}_{1}, \zeta_{1} ; T\right) & \equiv\left\langle\mathbf{q}_{2}, \zeta_{2}\left|e^{-(i / \hbar) \hat{H} T}\right| \mathbf{q}_{1}, \zeta_{1}\right\rangle \\
& =\int \frac{\mathcal{D}[\mathbf{q}] \mathcal{D}[\mathbf{p}]}{(2 \pi \hbar)^{d}} \mathcal{D} \mu[\zeta] \exp \left\{\frac{i}{\hbar} \mathcal{W}[\mathbf{q}, \mathbf{p}, \zeta ; T]\right\} .
\end{aligned}
$$

The integration is performed over the paths $[\mathbf{q}(t), \mathbf{p}(t), \zeta(t)]$ in the spin-orbit phase space connecting $\left(\mathbf{q}_{1}, \mathbf{p}_{1}, \zeta_{1}\right)$ to $\left(\mathbf{q}_{2}, \mathbf{p}_{2}, \zeta_{2}\right)$ in time $T$ with arbitrary $\mathbf{p}_{1}$ and $\mathbf{p}_{2}$. The integration measures are defined by

$$
\frac{\mathcal{D}[\mathbf{q}] \mathcal{D}[\mathbf{p}]}{(2 \pi \hbar)^{d}} \mathcal{D} \mu[\zeta]=\lim _{n \rightarrow \infty} \prod_{j=1}^{n-1} \frac{d \mathbf{q}\left(t_{j}\right) d \mathbf{p}\left(t_{j}\right)}{(2 \pi \hbar)^{d}} d \mu\left(\zeta\left(t_{j}\right)\right),
$$

where $t_{j}=j T / n$. The Hamilton principal function $\mathcal{W}=\mathcal{W}^{0}$ $+\hbar s \mathcal{W}^{1}$ consists of two contributions: the usual classical part,

$$
\mathcal{W}^{0}[\mathbf{q}, \mathbf{p} ; T]=\int_{0}^{T} d t\left[\mathbf{p} \cdot \dot{\mathbf{q}}-H^{0}(\mathbf{q}, \mathbf{p})\right],
$$

and the spin-related part,

$$
\mathcal{W}^{1}[\mathbf{q}, \mathbf{p}, \zeta ; T]=\int_{0}^{T} d t\left[\frac{\zeta \dot{\zeta}^{*}-\zeta^{*} \dot{\zeta}}{i\left(1+|\zeta|^{2}\right)}-\mathbf{n}(\zeta) \cdot \mathbf{C}(\mathbf{q}, \mathbf{p})\right]
$$

Now we can separate the integration over the spin paths in Eq. (4), thereby representing the propagator as ${ }^{56}$

$$
\begin{aligned}
U\left(\mathbf{q}_{2}, \zeta_{2}, \mathbf{q}_{1}, \zeta_{1} ; T\right)= & \int \frac{\mathcal{D}[\mathbf{q}] \mathcal{D}[\mathbf{p}]}{(2 \pi \hbar)^{d}} K_{[\mathbf{q}, \mathbf{p}]}\left(\zeta_{2}, \zeta_{1} ; T\right) \\
& \times \exp \left\{\frac{i}{\hbar} \mathcal{W}^{0}[\mathbf{q}, \mathbf{p} ; T]\right\}
\end{aligned}
$$

with

$$
K_{[\mathbf{q}, \mathbf{p}]}\left(\zeta_{2}, \zeta_{1} ; T\right)=\int \mathcal{D} \mu[\zeta] \exp \left\{i s \mathcal{W}^{1}[\mathbf{q}, \mathbf{p}, \zeta ; T]\right\} .
$$

Clearly, $K_{[\mathbf{q}, \mathbf{p}]}\left(\zeta_{2}, \zeta_{1} ; T\right)$ is a propagator of a system with the time-dependent Hamiltonian $\hat{H}_{[\mathbf{q}, \mathbf{p}]}(t)=\hbar \hat{\mathbf{s}} \cdot \mathbf{C}_{[\mathbf{q}, \mathbf{p}]}(t)$, where $\mathbf{C}_{[\mathbf{q}, \mathbf{p}]}(t)=\mathbf{C}(\mathbf{q}(t), \mathbf{p}(t))$ is calculated along the path $[\mathbf{q}(t), \mathbf{p}(t)]$ of the integral (8). This Hamiltonian describes a spin, precessing in the time-dependent magnetic field $\mathbf{C}_{[\mathbf{q}, \mathbf{p}]}(t){ }^{82}$ Expression (9) for $K_{[\mathbf{q}, \mathbf{p}]}\left(\zeta_{2}, \zeta_{1} ; T\right)$ can be inte- 
grated explicitly, ${ }^{60}$ yielding the usual spin propagator in the basis of coherent states (Appendix A).

We proceed by evaluating the path integral (8) in the semiclassical limit $\mathcal{W}^{0} \gg \hbar$. The integration simplifies considerably, if the spin-dependent Hamiltonian is treated as a perturbation, i.e., when

$$
\hbar s|\mathbf{C}(\mathbf{q}, \mathbf{p})| \ll\left|H^{0}(\mathbf{q}, \mathbf{p})\right| .
$$

This condition, assumed for the rest of the paper, is usually fulfilled in experiments based on the semiconductor heterostructures. According to this requirement, the spin-precession length must be much larger than the Fermi wavelength, however it can be smaller, of order, or greater than the system size. The semiclassical and perturbative regimes can be implemented simultaneously ${ }^{62,63}$ by formally letting $\hbar \rightarrow 0$ and keeping all other quantities fixed. Then the phase of the integrand in Eq. (8) is a rapidly varying functional, which justifies the use of the stationary-phase approximation. It is crucial that $K_{[\mathbf{q}, \mathbf{p}]}\left(\zeta_{2}, \zeta_{1} ; T\right)$ does not depend on $\hbar$, i.e., it is a slowly varying functional, and, therefore, its effect on the stationary trajectories can be neglected. Thus, the stationary trajectories are the extremals solely of $\mathcal{W}^{0}[\mathbf{q}, \mathbf{p} ; T]$, which means that they are the classical orbits of the spinless Hamiltonian $H^{0}$. The resulting semiclassical propagator,

$$
\begin{aligned}
& U_{\mathrm{sc}}\left(\mathbf{q}_{2}, \zeta_{2}, \mathbf{q}_{1}, \zeta_{1} ; T\right) \\
& \quad=\sum_{\gamma} K_{\gamma}\left(\zeta_{2}, \zeta_{1} ; T\right) \mathcal{C}_{\gamma} \exp \left\{\frac{i}{\hbar} \mathcal{W}_{\gamma}^{0}\left(\mathbf{q}_{2}, \mathbf{q}_{1} ; T\right)\right\},
\end{aligned}
$$

is a sum over all classical trajectories $\gamma \equiv\left[\mathbf{q}_{\gamma}(t), \mathbf{p}_{\gamma}(t)\right]$ of time $T$ from $\mathbf{q}_{1}$ to $\mathbf{q}_{2}$. The prefactor $\mathcal{C}_{\gamma}$, arising from the stationary-phase integration, is the same as in the spinless case: ${ }^{58}$

$$
\mathcal{C}_{\gamma}=\frac{\exp \left(-i \frac{\pi}{4} d-i \frac{\pi}{2} \nu_{\gamma}\right)}{(2 \pi \hbar)^{d / 2}}\left|\operatorname{det}_{\alpha \beta} \frac{\partial^{2} \mathcal{W}_{\gamma}^{0}\left(\mathbf{q}_{2}, \mathbf{q}_{1} ; T\right)}{\partial q_{2}^{\alpha} \partial q_{1}^{\beta}}\right|^{1 / 2},
$$

where $\nu_{\gamma}$ is the Maslov index. Although the classical trajectories are not affected by the spin motion, the reverse is not true. Indeed, the spin propagator $K_{\gamma}$, computed along the classical trajectories, describes the spin evolution in the effective magnetic field $\mathbf{C}_{\gamma}(t)=\mathbf{C}\left(\mathbf{q}_{\gamma}(t), \mathbf{p}_{\gamma}(t)\right)$ generated by these trajectories.

The semiclassical Green function is given by the Laplace transform of $U_{\mathrm{sc}}(T)$ to the energy domain $E$,

$$
G(E)=-\frac{i}{\hbar} \int_{0}^{\infty} d T e^{i\left(E+i 0^{+}\right) T / \hbar} U_{\mathrm{sc}}(T),
$$

evaluated in the stationary-phase approximation. As before, $K_{\gamma}(T)$ does not modify the stationary-phase condition, and the theory without spin can be applied. Moreover, using the resolution of unity (2), the spin propagator can be transformed to the usual $|\sigma\rangle$ basis. Finally, we obtain

$$
G_{\sigma^{\prime} \sigma}\left(\mathbf{q}_{2}, \mathbf{q}_{1} ; E\right)=\sum_{\gamma}\left(\hat{K}_{\gamma}\right)_{\sigma^{\prime} \sigma} \mathcal{F}_{\gamma} \exp \left\{\frac{i}{\hbar} \mathcal{S}_{\gamma}^{0}\left(\mathbf{q}_{2}, \mathbf{q}_{1} ; E\right)\right\}
$$

with $\sigma, \sigma^{\prime}=-s, \ldots, s$. In Eq. (14), $\gamma$ is a classical trajectory of the Hamiltonian $H^{0}=E$ with the action $\mathcal{S}_{\gamma}^{0}=\int_{\gamma} \mathbf{p} \cdot d \mathbf{q}$ and time $T_{\gamma}(E)=\partial \mathcal{S}_{\gamma}^{0} / \partial E . \hat{K}_{\gamma}(t)$ is the operator form of the spin propagator [Appendix A, Eqs. (A5) and (A6)], and $\left(\hat{K}_{\gamma}\right)_{\sigma^{\prime} \sigma}$ $\equiv\left\langle\sigma^{\prime}\left|\hat{K}_{\gamma}\left(T_{\gamma}(E)\right)\right| \sigma\right\rangle$ is its matrix element. The prefactor is given by

$$
\mathcal{F}_{\gamma}=\mathcal{C}_{\gamma} e^{-i(\pi / 4) \operatorname{sgn}\left(d T_{\gamma} / d E\right)},
$$

and $\mathcal{C}_{\gamma}$ is expressed in terms of the derivatives of $\mathcal{S}_{\gamma}^{0}\left(\mathbf{q}_{2}, \mathbf{q}_{1} ; E\right)$ (Ref. 58).

\section{SEMICLASSICAL LANDAUER FORMULA WITH SPIN}

The semiclassical Landauer formula with spin, derived below, is the main analytical result of this paper. It forms the basis for the subsequent semiclassical treatment of the spindependent transport in two-dimensional systems.

\section{A. Derivation of the formula}

We start from the standard (quantum) Landauer formula, that relates the conductance $\left(e^{2} / h\right) \mathcal{T}$ of a sample with two ideal leads to its transmission coefficient $\mathcal{T}$ (Ref. 64). Assuming that the leads support $N$ and $N^{\prime}$ open channels (not counting the spin degeneracy), respectively, the transmission can be expressed as the sum

$$
\mathcal{T}=\sum_{n=1}^{N^{\prime}} \sum_{m=1}^{N} \sum_{\sigma^{\prime}, \sigma=-s}^{s}\left|t_{n \sigma^{\prime}, m \sigma}\right|^{2} .
$$

Here $t_{n \sigma^{\prime}, m \sigma}$ is the transmission amplitude between the incoming channel $|m \sigma\rangle$ (with spin projection $\sigma$ ) and the outgoing channel $\left|n \sigma^{\prime}\right\rangle$ belonging to different leads. We shall also consider the reflection coefficient

$$
\mathcal{R}=\sum_{n, m=1}^{N} \sum_{\sigma^{\prime}, \sigma=-s}^{s}\left|r_{n \sigma^{\prime}, m \sigma}\right|^{2},
$$

where the reflection amplitude $r_{n \sigma^{\prime}, m \sigma}$ is defined for the channels of the same lead. The transmission and reflection satisfy the normalization condition

$$
\mathcal{T}+\mathcal{R}=(2 s+1) N
$$

that follows from the unitarity of the scattering matrix.

Consider, as a model for a (large) quantum dot, a twodimensional cavity (billiard) with hard-wall leads. The particle in the cavity is subjected to the SO and Zeeman interaction of the form (3). Semiclassical expressions for the transition amplitudes in the spinless case were derived in Refs. 48 and 49 by projecting a semiclassical Green function onto the lead eigenstates, while integrating over the lead cross sections in the stationary-phase approximation. For a particle with spin we implement this procedure using the 
semiclassical Green function (14). In the semiclassical limit of large action, $\mathcal{S}_{\gamma}^{0} \gg \hbar$, the spin-propagator element $\left(\hat{K}_{\gamma}\right)_{\sigma^{\prime} \sigma}$ does not shift the stationary point. In the resulting expression,

$$
t_{n \sigma^{\prime}, m \sigma}=\sum_{\gamma(\bar{n}, \bar{m})}\left(\hat{K}_{\gamma}\right)_{\sigma^{\prime} \sigma} \mathcal{A}_{\gamma} \exp \left(\frac{i}{\hbar} \mathcal{S}_{\gamma}\right)
$$

the only effect of spin is to weight the contribution of each trajectory in the sum with the respective matrix element of $\hat{K}_{\gamma}$. In Eq. (19) $\gamma(\bar{n}, \bar{m})$ is any classical trajectory of energy $E$ that enters (exits) the cavity at a certain angle $\Theta_{\bar{m}}\left(\Theta_{\bar{n}}\right)$ measured from the normal at a lead's cross section. ${ }^{83}$ The angles are determined by the transverse momentum in the leads: $\sin \Theta_{\bar{m}}=\bar{m} \pi / k w$ and $\sin \Theta_{\bar{n}}=\bar{n} \pi / k w^{\prime}$, where $k$ is the wave number and $w$ and $w^{\prime}$ are the widths of the entrance and exit leads. The action for a trajectory of length $L_{\gamma}$ is $\mathcal{S}_{\gamma}=\hbar k L_{\gamma}$. The prefactor is given by

$$
\begin{aligned}
\mathcal{A}_{\gamma}= & -\sqrt{\frac{\pi \hbar}{2 w w^{\prime}}} \frac{\operatorname{sgn}(\bar{n}) \operatorname{sgn}(\bar{m})}{\left|\cos \Theta_{\bar{n}} \cos \Theta_{\bar{m}} M_{21}^{\gamma}\right|^{1 / 2}} \\
& \times \exp \left[i k\left(\sin \Theta_{\bar{m}} y-\sin \Theta_{\bar{n}} y^{\prime}\right)-i \frac{\pi}{2}\left(\mu_{\gamma}-\frac{1}{2}\right)\right],
\end{aligned}
$$

where $M_{21}^{\gamma}$ is an element of the stability matrix (as defined, e.g., in Ref. 65), $y\left(y^{\prime}\right)$ is the coordinate on the lead's cross section at which the orbit $\gamma$ enters (exits) the cavity, and $\mu_{\gamma}$ is the modified Morse index. ${ }^{49}$ Substituting the sum (19) and the corresponding result for $r_{n \sigma^{\prime}, m \sigma}$ in Eqs. (16) and (17) we obtain the semiclassical approximation for the total transmission and reflection,

$$
(\mathcal{T}, \mathcal{R})=\sum_{n m} \sum_{\gamma(\bar{n}, \bar{m})} \sum_{\gamma^{\prime}(\bar{n}, \bar{m})} \mathcal{M}_{\gamma, \gamma^{\prime}} \mathcal{A}_{\gamma} \mathcal{A}_{\gamma^{\prime}}^{*} \exp \left(\frac{i}{\hbar}\left(\mathcal{S}_{\gamma}-\mathcal{S}_{\gamma^{\prime}}\right)\right) .
$$

Here in the case of transmission (reflection) the paths $\gamma$ and $\gamma^{\prime}$ connect different leads (return to the same lead). In this expression each orbital contribution is weighted with the spin modulation factor

$$
\mathcal{M}_{\gamma, \gamma^{\prime}} \equiv \operatorname{Tr}\left(\hat{K}_{\gamma} \hat{K}_{\gamma^{\prime}}^{\dagger}\right)
$$

where the trace is taken in spin space. Equations (21) and (22) generalize the semiclassical Landauer formula ${ }^{48,49}$ to the case of spin-dependent transport.

\section{B. Leading semiclassical contributions for a spinless particle}

In the semiclassical limit the phases in Eq. (21) are rapidly varying functions of energy, unless $\gamma$ and $\gamma^{\prime}$ have equal or nearly equal actions. Therefore, if one calculates the transmission and reflection averaged over a small energy window, most of the terms in the double sum will vanish. In the following, we review the leading contributions for a spinless system $\left(\mathcal{M}_{\gamma, \gamma^{\prime}} \equiv 1\right)$ with time-reversal symmetry:

(i) The classical part consists of the terms with $\gamma^{\prime}=\gamma$
(Ref. 66). Their fast-varying phases cancel (including the phase in the prefactor). For a classically ergodic (in particular, chaotic) system one finds ${ }^{50}$

$$
\mathcal{T}_{\mathrm{cl}}^{(0)}=\frac{N N^{\prime}}{N+N^{\prime}}, \quad \mathcal{R}_{\mathrm{cl}}^{(0)}=\frac{N^{2}}{N+N^{\prime}}
$$

(the superscript refers to zero spin and zero magnetic field). This result can be obtained using the sum rule ${ }^{50}$

$$
\sum_{\gamma(\bar{n}, \bar{m})}\left|\mathcal{A}_{\gamma}\right|^{2} \delta\left(L-L_{\gamma}\right) \simeq\left(N+N^{\prime}\right)^{-1} P_{L}(L) .
$$

It implies that the length $L$ of the classical trajectories is distributed according to

$$
P_{L}(L) \simeq \frac{1}{L_{\mathrm{esc}}} \exp \left(-\frac{L}{L_{\mathrm{esc}}}\right),
$$

in other words, the probability for a particle to stay in an open chaotic cavity decreases exponentially with time. The average escape length is given by

$$
L_{\mathrm{esc}}=\frac{\pi \mathrm{A}_{c}}{w+w^{\prime}}=\frac{k \mathrm{~A}_{c}}{N+N^{\prime}},
$$

where $\mathrm{A}_{c}$ is the area of the cavity. It is assumed that $L_{\mathrm{esc}}$ $\gg L_{b}$, where

$$
L_{b}=\pi \mathrm{A}_{c} / \mathrm{P}_{c}
$$

is the average distance between two consecutive bounces at the boundaries ${ }^{67}$ and $\mathrm{P}_{c}$ is the perimeter. In an arbitrary billiard the last expression is true if the average is taken over the ensemble of chords with random initial position and boundary component of the velocity. In ergodic billiards the average can, alternatively, be calculated along almost any trajectory.

(ii) The diagonal quantum correction is defined for reflection only. It contains the terms with $n=m$ and $\gamma^{\prime}=\gamma^{-1}$, where $\gamma^{-1}$ is the time reversal of $\gamma$ (Ref. 49) ${ }^{84}$ Clearly, when $n$ and $m$ are different channels, the orbits $\gamma(\bar{n}, \bar{m})$ and $\gamma^{\prime}(\bar{n}, \bar{m})$ cannot be the mutual time reversals, since reversing the time would exchange $\bar{n}$ and $\bar{m}$. Again, the two actions are equal, and the result for an ergodic system without spin reads $^{50}$

$$
\delta \mathcal{R}_{\text {diag }}^{(0)}=\frac{N}{N+N^{\prime}} .
$$

(iii) The loop contribution consists of pairs of long orbits that stay close to each other in the configuration space. One orbit of the pair has a self-crossing with a small crossing angle $\varepsilon$, thus forming a loop, its counterpart has an anticrossing. ${ }^{77,85}$ Away from the crossing region the orbits are located exponentially close to each other, they are related by time reversal along the loop and coincide along the tails $^{50,68}$ (Fig. 1). The action difference for these orbits is of second order in $\varepsilon$. For spinless chaotic systems with hyperbolic dynamics the loop terms in Eq. (21) yield ${ }^{50}$

$$
\delta \mathcal{T}_{\text {loop }}^{(0)}=-\frac{N N^{\prime}}{\left(N+N^{\prime}\right)^{2}}, \quad \delta \mathcal{R}_{\text {loop }}^{(0)}=-\frac{N(N+1)}{\left(N+N^{\prime}\right)^{2}} .
$$




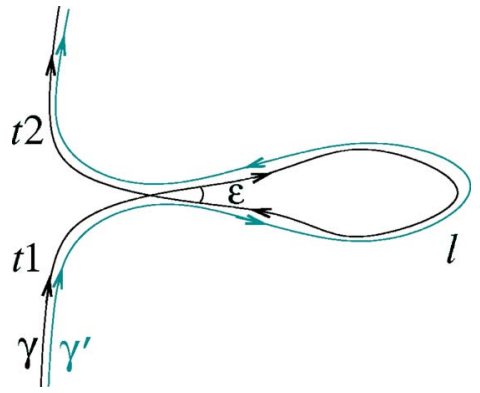

FIG. 1. (Color online) Pair of orbits with a loop. (Neglecting the crossing region, we distinguish between the tails $t 1, t 2$, and the loop l.)

From here on, we will work in the limit $N, N^{\prime} \gg 1$. In this semiclassical regime (in the leads) the classical contribution (23) (of the order $N$ ) is much greater than the quantum corrections (28) and (29) (of the order $N^{0}$ ), while higher-order loop corrections (of order $N^{-1}$ and smaller) can be neglected. We note that the normalization is preserved order by order,

$$
\begin{gathered}
\mathcal{T}_{\mathrm{cl}}^{(0)}+\mathcal{R}_{\mathrm{cl}}^{(0)}=N, \\
\delta \mathcal{R}_{\text {diag }}^{(0)}+\delta \mathcal{R}_{\text {loop }}^{(0)}+\delta \mathcal{T}_{\text {loop }}^{(0)}=\mathcal{O}\left(N^{-1}\right) .
\end{gathered}
$$

\section{Spin-dependent quantum corrections to transmission and reflection}

We now compute the spin modulation factor for the leading contributions to the energy-smoothed $\mathcal{T}$ and $\mathcal{R}$, identified in Sec. III B. First, the case with time-reversal symmetry ${ }^{86}$ is considered.

(i) For the classical part we find, using the unitarity of $\hat{K}_{\gamma}$, that the modulation factor $\mathcal{M}_{\gamma, \gamma}=\operatorname{Tr}\left(\hat{K}_{\gamma} \hat{K}_{\gamma}^{\dagger}\right)=2 s+1$ reduces to the trivial spin degeneracy.

(ii) For the diagonal quantum correction the result is

$$
\mathcal{M}_{\gamma, \gamma^{-1}}=\operatorname{Tr}\left(\hat{K}_{\gamma}^{2}\right) .
$$

It was taken into account that $\hat{K}_{\gamma^{-1}}^{\dagger}=\hat{K}_{\gamma}$, which follows from the relation $\mathbf{C}_{\gamma^{-1}}(t)=-\mathbf{C}_{\gamma}\left(T_{\gamma}-t\right)$ and Eq. (A5).

(iii) Assuming that the trajectories forming a loop pair (Fig. 1) coincide along the tails $t 1, t 2$ and are mutually timereversed along the loop $l$, thereby neglecting the crossing region, we can represent the propagators as $\hat{K}_{\gamma}=\hat{K}_{t 2} \hat{K}_{l} \hat{K}_{t 1}$ and $\hat{K}_{\gamma^{\prime}}=\hat{K}_{t 2} \hat{K}_{l^{-1}} \hat{K}_{t 1}$. Hence, the modulation factor

$$
\mathcal{M}_{\gamma, \gamma^{\prime}}=\operatorname{Tr}\left(\hat{K}_{l}^{2}\right) .
$$

is independent of the tails.

In the presence of a magnetic field the time-reversal symmetry is broken, and the preceding results should be adjusted. In this paper we consider a constant, uniform, arbitrarily directed magnetic field $\mathbf{B}$. Its component $B_{z}$ normal to the cavity is assumed to be weak enough, ${ }^{87}$ so as not to change the classical trajectories in Eq. (21), but only modify the action difference by the Aharonov-Bohm $(\mathrm{AB})$ phase. We define the $\mathrm{AB}$ modulation factor

$$
\varphi_{\gamma, \gamma^{\prime}} \equiv \exp \left(\frac{i}{\hbar} \Delta\left(\mathcal{S}_{\gamma}-\mathcal{S}_{\gamma^{\prime}}\right)\right)=\exp \left(i \frac{4 \pi \mathrm{A} B_{z}}{\Phi_{0}}\right) .
$$

Here, for a pair of trajectories $\gamma$ and $\gamma^{\prime}$ from the diagonal (loop) contribution, $\mathrm{A} \equiv \int_{\gamma(l)} \mathbf{A} \cdot d \mathbf{l} / B_{z}$ is the effective enclosed area, where the integral of the vector potential $\mathbf{A}$ is taken along $\gamma$ (its loop part $l$ ), and $\Phi_{0}=h c / e$ is the flux quantum.

With the Zeeman interaction included, Eqs. (32) and (33) for the spin modulation factor are no longer valid. In fact, if we distinguish the $\mathrm{SO}$ and Zeeman terms in the effective magnetic field $\mathbf{C}_{\gamma}(t)=\mathbf{C}_{\gamma}^{\mathrm{SO}}(t)+\mathbf{C}_{\gamma}^{Z}(t)$, the diagonal (and, correspondingly, the loop) modulation factor can be written in the form

$$
\mathcal{M}_{\gamma, \gamma^{-1}}(\mathbf{B})=\operatorname{Tr}\left(\hat{K}_{\gamma} \hat{K}_{\tilde{\gamma}}\right)
$$

where $\tilde{\gamma}$ is a fictitious trajectory producing the field $\mathbf{C}_{\tilde{\gamma}}(t)$ $=\mathbf{C}_{\gamma}^{\mathrm{SO}}(t)-\mathbf{C}_{\gamma}^{Z}(t)$. Clearly, in the absence of SO coupling, we have $\mathcal{M}_{\gamma, \gamma^{-1}}(\mathbf{B})=2 s+1$, i.e., the Zeeman field alone does not affect the modulation factor.

In Refs. 49 and 50 the quantum corrections to transmission and reflection in the presence of magnetic field were calculated for an ergodic system. We extend their approach to a system with SO interaction. To this end, we consider the generalized modulation factor

$$
(\mathcal{M} \varphi)_{\gamma, \gamma^{\prime}} \equiv \mathcal{M}_{\gamma, \gamma^{\prime}} \varphi_{\gamma, \gamma^{\prime}}
$$

The diagonal contribution can be computed using the sum rule (24). First, one averages $(\mathcal{M} \varphi)_{\gamma, \gamma^{\prime}}$ for the time-reversed pairs of trajectories and loops of a given length $L$. Thus, the average is performed over the ensemble of almost closed orbits. This restriction proves very important, since the average modulation factor for closed and open trajectories is different (see Sec. V). The average modulation factor $\overline{\mathcal{M} \varphi}(L ; \mathbf{B})$ is then further weighted with the length distribution (25). It can be shown that for the loop contribution in a hyperbolic system with a single Lyapunov exponent holds effectively the same procedure (see Ref. 50 and Appendix B). Hence, the diagonal and the loop relative quantum corrections to reflection and transmission are equal and given by

$$
\begin{aligned}
\frac{\delta \mathcal{R}_{\text {diag }}(\mathbf{B})}{\delta \mathcal{R}_{\text {diag }}^{(0)}} & =\frac{\delta \mathcal{R}_{\text {loop }}(\mathbf{B})}{\delta \mathcal{R}_{\text {loop }}^{(0)}}=\frac{\delta \mathcal{T}_{\text {loop }}(\mathbf{B})}{\delta \mathcal{T}_{\text {loop }}^{(0)}}=\langle\overline{\mathcal{M} \varphi}(\mathbf{B})\rangle_{L} \\
& \equiv \frac{1}{L_{\text {esc }}} \int_{0}^{\infty} d L e^{-L / L_{\text {esc }} \overline{\mathcal{M} \varphi}(L ; \mathbf{B})}
\end{aligned}
$$

The normalization condition (18) is preserved due to Eq. (31).

When the SO and Zeeman interactions are absent, the average modulation factor $(2 s+1) \bar{\varphi}(L ; \mathbf{B})$ in a chaotic system can be analytically estimated using the Gaussian distribution of enclosed areas ${ }^{49}$

$$
P_{\mathrm{A}}(\mathrm{A} ; L) \simeq \frac{1}{\sqrt{2 \pi \mathrm{A}_{0}^{2} L / L_{b}}} \exp \left(-\frac{\mathrm{A}^{2}}{2 \mathrm{~A}_{0}^{2} L / L_{b}}\right) .
$$

It depends on a system-specific parameter $A_{0}$, a typical area enclosed by an orbit during one circulation. This distribution 
does not depend on the incoming and outgoing channel numbers and is valid for both closed and open trajectories. The average (37) yields ${ }^{49,50}$

$$
\langle\overline{\mathcal{M} \varphi}(\mathbf{B})\rangle_{L}=\frac{2 s+1}{1+\widetilde{B}^{2} L_{\mathrm{esc}} / L_{b}},
$$

where $\widetilde{B}=2 \sqrt{2} \pi B_{z} \mathrm{~A}_{0} / \Phi_{0}$. Thus the quantum corrections have a Lorentzian dependence on the magnetic field. The result (39) is specific to chaotic systems as it depends on $L_{\text {esc }}$ - such a parameter is not relevant to extended disordered systems, while for regular billiards $P_{L}(L)$ (introduced earlier) is usually a power law. ${ }^{49}$ The increase of reflection (decrease of transmission) for $B_{z}=0$ constitutes the effect of weak localization. A magnetic field destroys the time-reversal symmetry and, thereby, the interference between the mutually time-reversed and loop pairs of paths, thus diminishing the quantum corrections.

The SO interaction may turn the constructive interference between the orbit pairs into destructive one. Since the sign of the quantum corrections in this case would be reversed, one speaks of weak antilocalization. In the following sections we study the transition from WL to WA and the related question of spin relaxation.

\section{SPIN RELAXATION}

\section{A. General discussion}

Equation (37) demonstrates that the modulation factor $\overline{\mathcal{M} \varphi}(L ; \mathbf{B})$ is a key to calculating the quantum corrections to the conductance. Hence, we will first examine $\overline{\mathcal{M} \varphi}$ in detail. As a function of length, this quantity contains information about the average spin evolution along the trajectories of the system. Here, by the spin evolution along a trajectory $\gamma$ we mean the change of the spin propagator $\hat{K}_{\gamma}(t)$. According to Appendix A, it can be written in the form [Eq. (A6)]

$$
\hat{K}_{\gamma}(t)=\exp \left[-i \hat{\mathbf{s}} \cdot \boldsymbol{\eta}_{\gamma}(t)\right]
$$

and, thus, depends on three real parameters, the rotation angle $\eta_{\gamma}(t)$ and the rotation axis given by the unit vector $\mathbf{m}_{\gamma}(t) \equiv \boldsymbol{\eta}_{\gamma}(t) / \eta_{\gamma}(t)$. Alternatively, one can parametrize $\hat{K}_{\gamma}$ using the elements of the corresponding $\mathrm{SU}(2)$ matrix [Eq. (A3)],

$$
W_{\gamma}(t)=e^{-i \boldsymbol{\sigma} \cdot \boldsymbol{\eta}_{\gamma}(t) / 2}=\left(\begin{array}{cc}
a_{\gamma}(t) & b_{\gamma}(t) \\
-b_{\gamma}^{*}(t) & a_{\gamma}^{*}(t)
\end{array}\right),
$$

which are restricted by the condition det $W_{\gamma}=\left|a_{\gamma}\right|^{2}+\left|b_{\gamma}\right|^{2}=1$. The two parametrizations are related by Eq. (A4). Clearly, $W_{\gamma}$ is the matrix representation of $\hat{K}_{\gamma}$ for spin $s=1 / 2$. Instead of $W_{\gamma}(t)$, we can consider the evolution of the spinor $\psi_{\gamma}(t)$ $\equiv\left(a_{\gamma}(t),-b_{\gamma}^{*}(t)\right)^{T}$, starting from the spin-up state $\psi_{\gamma}(0)$ $=(1,0)^{T}$. It is characterized by the spin direction $\mathbf{n}_{\gamma}(t)$ $=\left[\psi_{\gamma}(t)\right]^{T} \boldsymbol{\sigma} \psi_{\gamma}(t)$ and the overall phase $(\boldsymbol{\sigma}$ is the vector of Pauli matrices). For spin $s>1 / 2$ these are the direction and the phase of a coherent state (see Sec. II). Note that $\mathbf{n}_{\gamma}(t)$ results from the rotation of $\mathbf{n}_{\gamma}(0)=(0,0,1)$ by the angle $\eta_{\gamma}(t)$ about $\mathbf{m}_{\gamma}(t)$. Sometimes it is convenient to represent $W_{\gamma}(t)$ by a trajectory on the three-dimensional unit sphere $S^{3}$. For this purpose we define a four-dimensional unit vector

$$
\begin{aligned}
\boldsymbol{\xi}_{\gamma}(t) & =\left(-\operatorname{Im} b_{\gamma}(t),-\operatorname{Re} b_{\gamma}(t),-\operatorname{Im} a_{\gamma}(t), \operatorname{Re} a_{\gamma}(t)\right) \\
& =\left(\mathbf{m}_{\gamma}(t) \sin \frac{\eta_{\gamma}(t)}{2}, \cos \frac{\eta_{\gamma}(t)}{2}\right) .
\end{aligned}
$$

The trajectory starts at the "north pole," i.e., $\boldsymbol{\xi}_{\gamma}(0)$ $=(0,0,0,1)$.

Using the propagator matrix (41), the modulation factor Eqs. (32) and (33) for spin-1/2 can be expressed as

$$
\mathcal{M}=\operatorname{Tr}\left(W^{2}\right)=4 \xi_{4}^{2}-2=2 \cos \eta,
$$

where $\xi_{4}$ is the fourth component of $\boldsymbol{\xi}$ (in Appendix $\mathrm{C}$ an arbitrary $s$ is considered). To simplify the notation, we dropped the subscripts labeling the trajectory, and the time $t$ is the trajectory or loop time.

For long orbits one expects that the spin state becomes completely randomized due to SO interaction, if the particle motion is irregular. This means that all points $\xi \in S^{3}$ are equally probable, and, on average, $\overline{\xi_{4}^{2}}=1 / 4$ in the limit $L$ $\rightarrow \infty$. Hence, for $\mathbf{B}=0$ the modulation factor $\overline{\mathcal{M}}(L)$ $\equiv \overline{\mathcal{M} \varphi}(L ; 0)$ changes with $L$ from the positive value $\overline{\mathcal{M}}(0)$ $=2$ to the negative asymptotic value $\overline{\mathcal{M}}(\infty)=-1$ (cf. Ref. 69). The stronger the SO interaction, the shorter the length scale $L_{\mathcal{M}}$ of this change. If $L_{\text {esc }} \ll L_{\mathcal{M}}$, i.e., the particle quickly leaves the cavity, or the SO interaction is weak, then the relative quantum corrections (37) are positive, giving rise to WL. In the opposite case of strong SO interaction or long dwell times, the relative quantum corrections are negative, leading to WA. For an arbitrary spin, $\overline{\mathcal{M}}(L)$ changes from $\overline{\mathcal{M}}(0)=2 s+1$ to $\overline{\mathcal{M}}(\infty)=(-1)^{2 s}$ (Appendix C). Thus, WA cannot be observed for an integer spin, at least, for $L_{\mathrm{esc}}$ $\gg L_{\mathcal{M}}[\overline{\mathcal{M}}(L)$ can, in principle, become negative at intermediate lengths].

For the rest of the paper we will consider the physically most important case of spin $s=1 / 2$. If the Zeeman interaction is included, then Eq. (35) yields

$$
\mathcal{M}(\mathbf{B})=4 \xi_{4} \widetilde{\xi}_{4}-2 \xi \cdot \widetilde{\xi}
$$

where $\widetilde{\boldsymbol{\xi}}$ belongs to the fictitious trajectory $\widetilde{\gamma}$. In the absence of Zeeman coupling, the vectors $\boldsymbol{\xi}$ and $\widetilde{\boldsymbol{\xi}}$ coincide. Then the negative second term in Eq. (44) is responsible for the WA, if the first term is, on average, small enough due to SO interaction. An admixture of a moderate Zeeman coupling destroys the correlation between $\boldsymbol{\xi}$ and $\widetilde{\boldsymbol{\xi}}$, thereby reducing the average product $\bar{\xi} \cdot \widetilde{\xi}$. Thus, an external magnetic field suppresses WA in two ways: the AB flux breaks down the constructive interference between the orbital phases and the Zeeman interaction affects the spin modulation factor. As we know, the former mechanism inhibits the WL, as well.

The spin propagator $\hat{K}_{\gamma}(t)$ can be used not only in the calculation of the quantum corrections (37) —it also provides information about the spin relaxation along classical trajec- 
tories, which is of separate interest. The relaxation of the spin direction can be described by the $z$ component of the vector $\mathbf{n}_{\gamma}$,

$$
n_{z}=2\left(\xi_{3}^{2}+\xi_{4}^{2}\right)-1
$$

The ensemble average $\overline{n_{z}}(L)$ varies from $\overline{n_{z}}(0)=1$ to $\overline{n_{z}}(\infty)$ $=0$, if the memory of the initial spin direction is completely lost for long orbits. The typical length scale of this decay can be different from $L_{\mathcal{M}}$, because $\overline{\mathcal{M}}(L)$ depends on the phase of the spin state, as well as on its direction. Moreover, the length scale of $\overline{n_{z}}(L)$, as defined by Eq. (45), depends on the choice of the quantization axis. An invariant measure of the spin relaxation is given by $\overline{\xi_{4}^{2}}(L)$ or, equivalently, by $\overline{\mathcal{M}}(L)$. The different relaxation rates of $\overline{n_{z}}(L)$ and $\overline{\mathcal{M}}(L)$ are observed in two-dimensional systems with the Rashba and the Dresselhaus SO coupling (see Secs. IV B and V).

\section{B. Example: Diffusive systems}

In three-dimensional extended diffusive conductors ${ }^{88}$ the directions of the effective magnetic field $\mathbf{C}_{\gamma}(t)$ before and after a scattering event can be assumed uncorrelated. ${ }^{30} \mathrm{We}$ model this by keeping $|\mathbf{C}|=$ const and changing the direction of $\mathbf{C}$ randomly at identical time intervals equal to the elastic scattering time $\tau$. The spin propagator for the $j$ th time interval is $\hat{K}_{j}=\exp \left(-i \hat{\mathbf{s}} \cdot \mathbf{m}_{j}|\mathbf{C}| \tau\right), j=1,2, \ldots$, where $\mathbf{m}_{j}$ is a random unit vector. The position on $S^{3}$ after the first time interval is, according to Eq. (42), $\boldsymbol{\xi}(\tau)=\left(\mathbf{m}_{1} \sin |\mathbf{C}| \tau / 2, \cos |\mathbf{C}| \tau / 2\right)$. Thus, a trajectory on the sphere, starting at the "north pole," traverses an arc of length $|\mathbf{C}| \tau / 2$ along a randomly chosen great circle. During the second time interval, the trajectory starts at $\boldsymbol{\xi}(\tau)$ and moves along another random great-circle segment, and so on. Clearly, $\boldsymbol{\xi}(t)$ follows a random walk on $S^{3}$. In the continuous limit $|\mathbf{C}| \tau \ll 2 \pi$, its probability density satisfies a diffusion equation. Solving this equation (Appendix D) we find that the average modulation factor for trajectories of time $t$,

$$
\overline{\mathcal{M}}_{\text {diff,3D }}(t)=3 e^{-(1 / 3)|\mathbf{C}|^{2} \pi t}-1,
$$

and the average spin polarization,

$$
{\overline{\left(n_{z}\right)}}_{\operatorname{diff}, 3 \mathrm{D}}(t)=e^{-(1 / 3)|\mathbf{C}|^{2} \pi},
$$

exhibit the same relaxation rate. Note that Eq. (37) is not valid in diffusive systems. The modulation factor (46) is equivalent to the result of Eq. (10.12) of Ref. 30.

In two-dimensional diffusive systems with Rashba or Dresselhaus interaction it is reasonable to assume that $\mathbf{C}$ acquires a random direction in a two-dimensional plane. In this case the walk on $S^{3}$ is not fully random. As our numerical simulations show (Fig. 2), the modulation factor is reasonably well described by Eq. (46). However, the off-plane polarization $\overline{n_{z}}(t)$ relaxes faster in two dimensions [cf. Eqs. (34) and (35) of Ref. 70]. This is not surprising, since, obviously, $\xi_{3}(\tau) \equiv 0$ in $2 \mathrm{D}$, but not in $3 \mathrm{D}$.

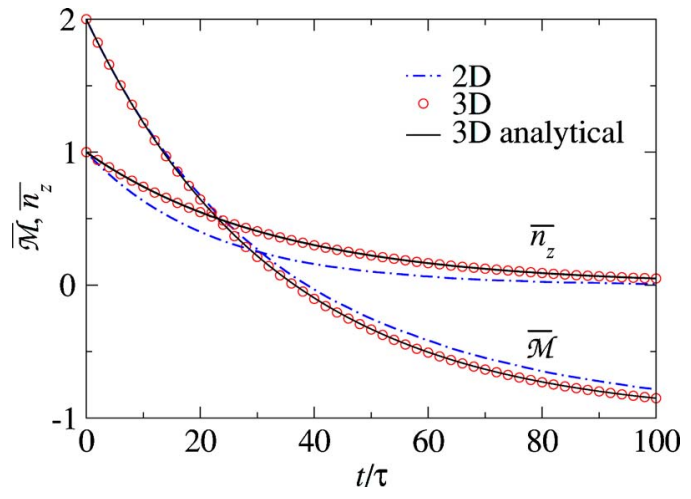

FIG. 2. (Color online) Average spin modulation factor $\overline{\mathcal{M}}(t)$ and spin projection $\overline{n_{z}}(t)$ in diffusive systems. The effective magnetic field $\mathbf{C}(t)$ changes its direction randomly at equal time intervals $\tau$. Its magnitude is kept constant and is equal to $0.3 / \tau$ in this example. The analytical expressions (46) and (47) (solid curves) are compared with the results of numerical simulations for $\mathbf{C}$ in two (dashed-dotted curves) and three (circles) dimensions. The average was performed over $10^{5}$ random sequences ("trajectories").

\section{RASHBA AND DRESSELHAUS INTERACTION: SPIN RELAXATION}

\section{A. Effective magnetic field}

We apply the general theory of the preceding sections to ballistic quantum dots with Rashba ${ }^{3}$ and Dresselhaus ${ }^{4}$ SO interaction. Both contributions are usually present in GaAs/AlGaAs heterostructures. Their strength ratio can be experimentally varied, e.g., by tuning the Rashba SO strength through an additional gate voltage. ${ }^{21}$ When the twodimensional electron gas lies in the (001) plane of a zincblende lattice, the effective magnetic field $\hat{\mathbf{C}}=\hat{\mathbf{C}}_{R}+\hat{\mathbf{C}}_{D}$ in the Hamiltonian (3) consists of

$$
\begin{gathered}
\hat{\mathbf{C}}_{R}=\frac{2 \pi}{\Lambda_{R}} \hat{\mathbf{v}} \times \mathbf{e}_{z}, \\
\hat{\mathbf{C}}_{D}=\frac{2 \pi}{\Lambda_{D}}\left(\hat{v}_{x} \mathbf{e}_{x}-\hat{v}_{y} \mathbf{e}_{y}\right),
\end{gathered}
$$

where the $x$ and $y$ axes are chosen along the [100] and [010] crystallographic directions, respectively, and $\hat{\mathbf{v}}=(\hat{\mathbf{p}}-e \mathbf{A} / c) /$ $M$ is the (Fermi-)velocity operator depending on the effective mass $M$. In Eq. (48) [Eq. (49)] the Rashba (Dresselhaus) interaction, usually characterized by the constant $\alpha_{R}\left(\alpha_{D}\right)$, is measured in terms of the inverse spin-precession length $\Lambda_{R(D)}^{-1}=\alpha_{R(D)} M / \pi \hbar^{2}$. In billiards the natural dimensionless parameter is

$$
\theta_{R(D)}=2 \pi L_{b} / \Lambda_{R(D)} .
$$

It signifies the mean spin-precession angle per bounce if only one type of SO interaction is present.

As can be seen from Eq. (48), the effective Rashba magnetic field $\mathbf{C}_{R}(t)$ generated by a particular trajectory points perpendicular to the velocity $\mathbf{v}(t)$. The directions of the Dresselhaus field $\mathbf{C}_{D}(t)$ and $\mathbf{v}(t)$ are symmetric with respect to the $x$ axis [Eq. (49)]. Hence, $\mathbf{C}_{R}(t)$ and $\mathbf{C}_{D}(t)$ always point 


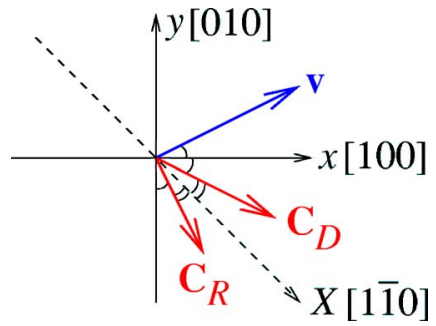

FIG. 3. (Color online) The Rashba effective field $\mathbf{C}_{R}$ is normal to the velocity $\mathbf{v}$, while the directions of the Dresselhaus field $\mathbf{C}_{D}$ and $\mathbf{v}$ are symmetric with respect to [100]. Thus, the directions of $\mathbf{C}_{R}$ and $\mathbf{C}_{D}$ are symmetric with respect to [1피.

symmetrically with respect to the $[1 \overline{1} 0]$ direction, labeled here by $X$ (Fig. 3). As a consequence, the total field $\mathbf{C}(t)$ is reflected about $X$ under the exchange $\Lambda_{R} \leftrightarrow \Lambda_{D}$ in Eqs. (48) and (49). This means that the modulation factor $\mathcal{M}(t)$ and the polarization projections $n_{z}(t)$ and $n_{X}(t)$ are preserved under this transformation. For example, systems with only Rashba or only Dresselhaus interaction have identical spin evolution, if the coupling strengths are the same.

It is sometimes convenient to work in the coordinate frame of $X$ and $Y=[110]$ (cf. Ref. 42). The projections of the effective magnetic field on these axes are given by

$$
\hat{C}_{X}=2 \pi \hat{v}_{Y} / \Lambda_{X}, \quad \hat{C}_{Y}=2 \pi \hat{v}_{X} / \Lambda_{Y},
$$

where

$$
\Lambda_{X}^{-1}=\Lambda_{D}^{-1}+\Lambda_{R}^{-1}, \quad \Lambda_{Y}^{-1}=\Lambda_{D}^{-1}-\Lambda_{R}^{-1}
$$

are the effective inverse precession lengths. As above, we can define dimensionless parameters $\theta_{X(Y)}=2 \pi L_{b} / \Lambda_{X(Y)}$.

\section{B. Numerical study}

The computation of the spin evolution in billiard cavities is relatively straightforward, since the classical trajectories there are sequences of straight segments. If only the uniform Rashba and Dresselhaus interaction is present, the effective magnetic field $\mathbf{C}_{j}$ is constant along the $j$ th segment (the segment velocity $\mathbf{v}_{j}$ is constant, moreover, its magnitude $v$ is the same for all $j$ due to the energy conservation). The spinpropagator matrix (41) for a trajectory,

$$
W \equiv e^{-i \boldsymbol{\sigma} \cdot \boldsymbol{\eta} / 2}=W_{l} \cdots W_{1},
$$

is a product of the respective matrices

$$
W_{j} \equiv e^{-i \boldsymbol{\sigma} \cdot \boldsymbol{\eta}_{j} / 2}=e^{-i \boldsymbol{\sigma} \cdot \mathbf{C}_{j} t_{j} / 2} \quad(j=1, \ldots, l)
$$

for the $l$ orbit segments. In practice, it is convenient to remove the velocity dependence in Eqs. (51) by using the displacement $\Delta \mathbf{r}_{j} \equiv \Delta X_{j} \mathbf{e}_{X}+\Delta Y_{j} \mathbf{e}_{Y}=\mathbf{v}_{j} t_{j}$ instead of the segment time $t_{j}$. Thereby the rotation vector can be expressed as

$$
\boldsymbol{\eta}_{j}=2 \pi\left(\frac{\Delta Y_{j}}{\Lambda_{X}} \mathbf{e}_{X}+\frac{\Delta X_{j}}{\Lambda_{Y}} \mathbf{e}_{Y}\right) .
$$

It follows from this equation that rescaling of the system size and the spin-precession lengths by the same factor does not

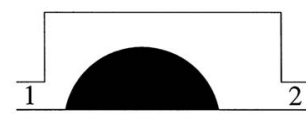

DS

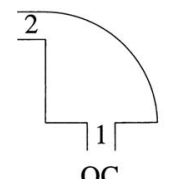

QC

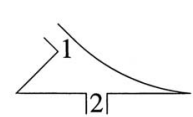

DD

rectangle

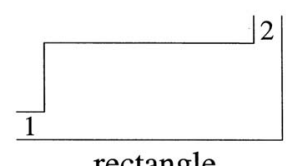

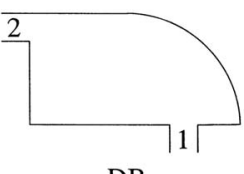

DB

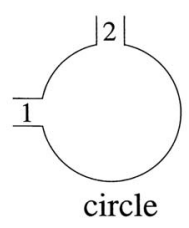

FIG. 4. Billiard geometries: desymmetrized Sinai (DS) billiard, desymmetrized diamond (DD) billiard, desymmetrized Bunimovich (DB) stadium billiard, quarter circle (QC), rectangle, and circle. The leads are numbered for future reference.

change the spin relaxation. In other words, given the shape of the billiard, the averages $\overline{n_{z}}$ and $\overline{\mathcal{M}}$ as functions of $L / L_{b}$ (computed below) depend only on the angles $\theta_{R}$ and $\theta_{D}$.

We performed a systematic numerical study of spin relaxation for several billiard geometries (Fig. 4) representative for systems with chaotic and integrable classical dynamics. The desymmetrized Sinai (DS) billiard, the desymmetrized diamond $^{71}$ (DD) billiard, and the desymmetrized Bunimovich (DB) stadium billiard represent chaotic cavities. The quarter circle $(\mathrm{QC})$, rectangle, and circle are integrable. The average spin relaxation is computed for the closed versions of these billiards.

Figures 5 and 6 depict the average spin relaxation described by $\overline{n_{z}}(L)$ and its invariant counterpart, $\overline{\mathcal{M}}(L)$, for the chaotic DS and integrable QC billiard. The average is per-

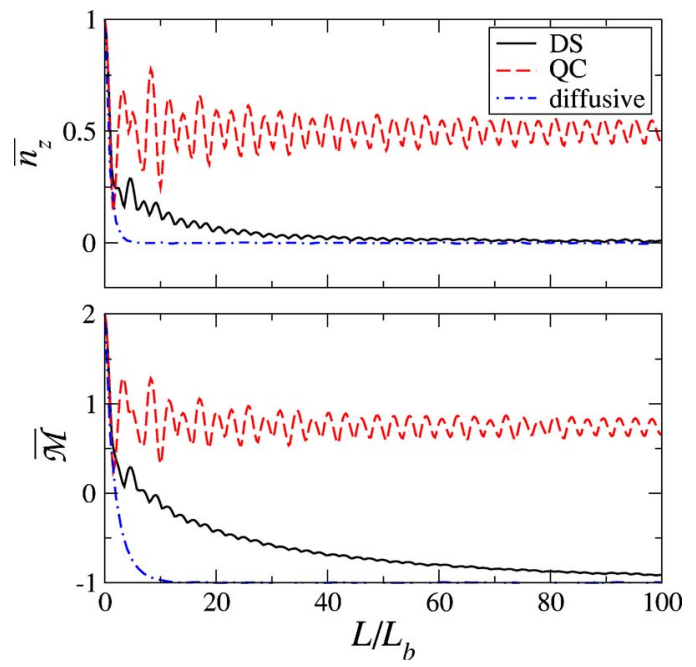

FIG. 5. (Color online) Average spin projection $\overline{n_{z}}(L)$ and modulation factor $\overline{\mathcal{M}}(L)$ for the closed chaotic DS (solid curves) and integrable QC (dashed curves) billiard (see Fig. 4). Each data point represents the average over 50000 open trajectories with random initial values at the boundary. The numerical results for a twodimensional extended diffusive system (dashed-dotted curves), where $L_{b}$ is identified with the elastic mean free path $v \tau$, are shown for comparison. The SO-coupling strength is $\theta_{R} / 2 \pi=0.2$ and $\theta_{D}$ $=0$. 


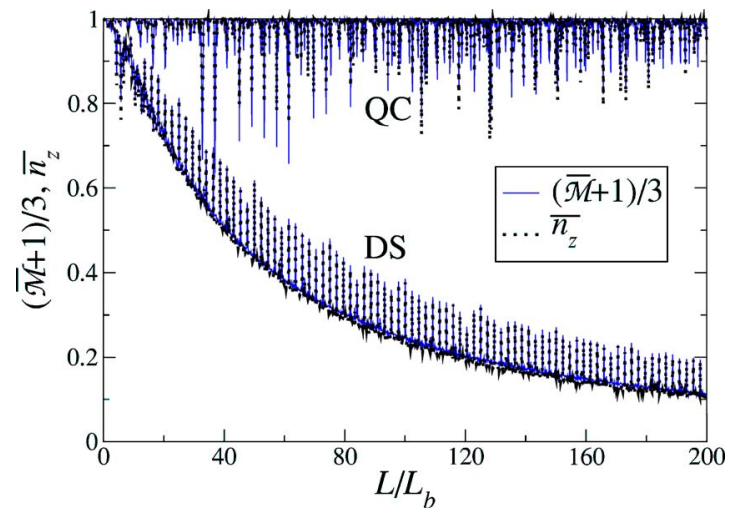

FIG. 6. (Color online) Average spin projection $\overline{n_{z}}(L)$ (dotted curves) and rescaled modulation factor $[\overline{\mathcal{M}}(L)+1] / 3$ (solid curves) for the closed chaotic DS and integrable QC billiard. Each data point represents the average over 5000 closed trajectories started at random at the boundary. The SO-coupling strength is $\theta_{R} / 2 \pi=0.2$ and $\theta_{D}=0$.

formed over ensembles of open (Fig. 5) and closed (Fig. 6) trajectories of length $L$ starting at random position at the boundary with a random boundary component of the velocity. The strength of the Rashba interaction is chosen as $\theta_{R} / 2 \pi=0.2$, and the Dresselhaus interaction is absent (or, equivalently, $\theta_{D} / 2 \pi=0.2$ and $\left.\theta_{R}=0\right)$.

In Fig. 5, the numerical results for an extended twodimensional diffusive system, where $L_{b}$ is identified with the elastic mean free path $v \tau$, are shown for comparison. We observe that on the scale of $L \sim L_{b}$ the spin relaxation is the same in all three examples. Indeed, before the first collision with the boundary or a scatterer, the particle moves along a straight line, irrespective of the system it belongs to. On longer length scales relaxation in an extended diffusive system is much stronger than in confined systems. Moreover, in the integrable billiard saturation takes place. ${ }^{89}$ We also note that $\bar{n}_{z}(L)$ in the chaotic billiard, similarly to the diffusive system (Sec. IV B), relaxes to its asymptotic value faster than $\overline{\mathcal{M}}(L)$.

The ensemble of closed orbits (Fig. 6) is responsible for the quantum corrections to transmission and reflection (Sec. III). We find that the relaxation in this case is much slower than for the ensemble of arbitrary trajectories. Remarkably, the spin projection $\bar{n}_{z}$ and the rescaled modulation factor $(\overline{\mathcal{M}}+1) / 3$ are hardly distinguishable.

The spin evolution in several chaotic systems is compared in Figs. 7 and 8 for the ensembles of open and closed trajectories, respectively. All the billiards show a qualitatively similar behavior. The DS and DD billiards have about the same relaxation rate. In the DB billiards the relaxation rate grows continuously, starting from zero, as the ratio of the upper straight side to the radius increases.

In Fig. 9 the modulation factor averaged over the open trajectories is presented for the integrable QC and rectangle billiard. Both systems are characterized by the saturation of spin relaxation and persistent long-time oscillations. The saturation level decreases down to -1 as the SO coupling becomes stronger. The circle billiard, which shows a non-

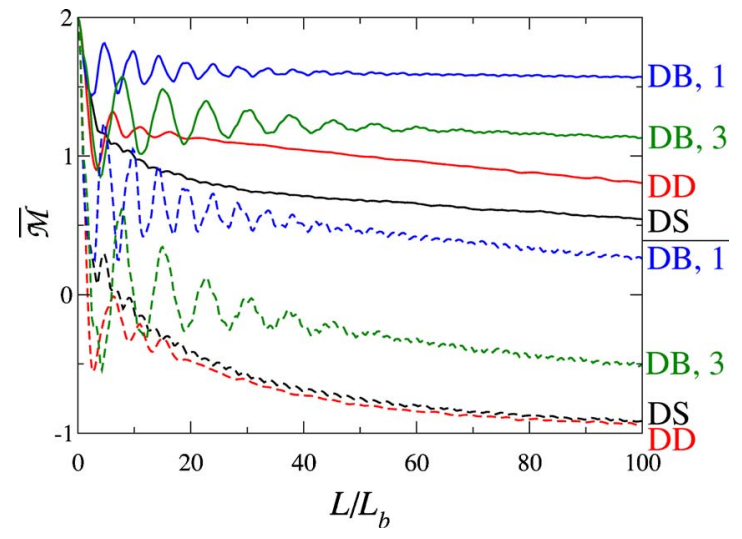

FIG. 7. (Color online) The average modulation factor $\overline{\mathcal{M}}(L)$ for the closed chaotic DS, DD, and DB billiards. In the latter case the ratio of the upper straight side to the radius was taken to be 1 (DB, 1) and $3(\mathrm{DB}, 3)$. Each data point represents the average over 50000 open trajectories started at random at the boundary. The SO-coupling strength is $\theta_{R} / 2 \pi=0.1$ (four upper curves) and 0.2 (four lower curves), while $\theta_{D}=0$.

typical relaxation pattern, is considered in Sec. V C.

When the Rashba and the Dresselhaus couplings work simultaneously ${ }^{78-80}$ they mutually counteract their effects on the spin relaxation ${ }^{90}$ (Fig. 10). In the extreme case $\Lambda_{R}=\Lambda_{D}$, i.e., $\Lambda_{Y}^{-1}=0$, the effective field $\mathbf{C}$ is always parallel to the $X$ axis $^{91}$ (Fig. 3). Hence, the propagator matrices in Eq. (53) commute, and the rotation vector becomes

$$
\boldsymbol{\eta}=\sum_{j=1}^{l} \boldsymbol{\eta}_{j}=2 \pi\left(\Delta Y / \Lambda_{X}\right) \mathbf{e}_{X},
$$

where $\Delta Y=\sum_{j=1}^{l} \Delta Y_{j}$ is the $Y$ displacement for the trajectory. According to Eq. (43), the modulation factor is then

$$
\mathcal{M}=2 \cos \left(2 \pi|\Delta Y| / \Lambda_{X}\right) .
$$

On the long-length scale $L \gg L_{b},|\Delta Y|$ varies from orbit to orbit between 0 and the system size. Clearly, the average $\overline{\mathcal{M}}$ should be independent of the orbit length $L$. This explains the saturation in Fig. 10. The saturation level decreases from 2 to 0 as $\theta_{R}$ changes from 0 to $\infty$. For closed orbits we have

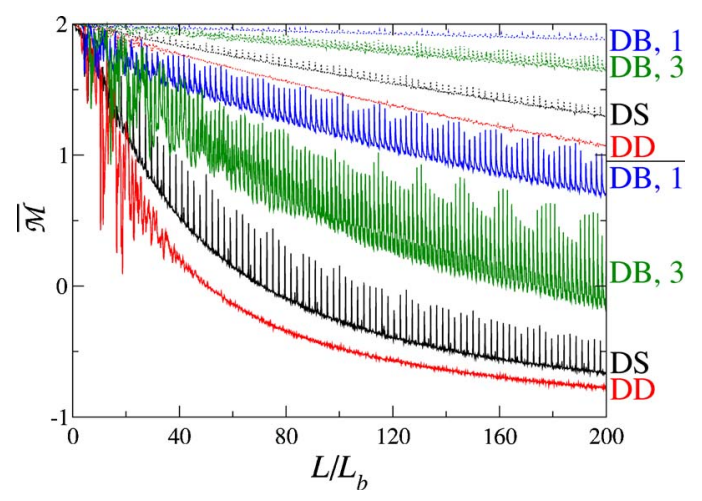

FIG. 8. (Color online) Same as in Fig. 7, but for the ensemble of closed orbits started at random at the boundary. Each data point represents the average over 5000 trajectories. 


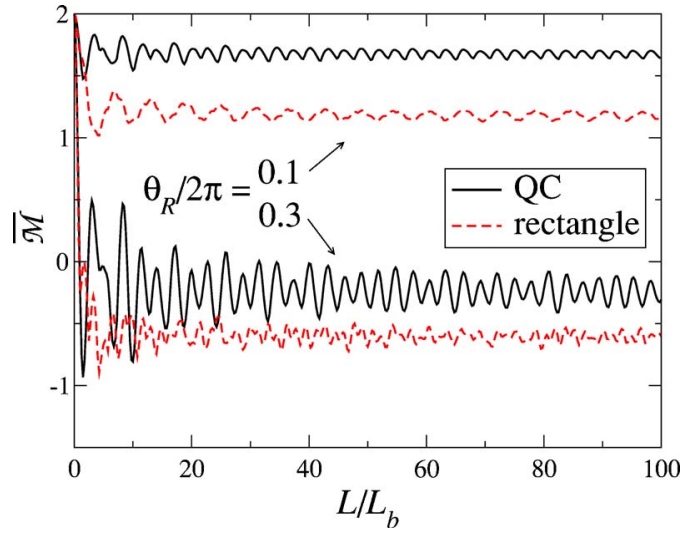

FIG. 9. (Color online) The average modulation factor $\overline{\mathcal{M}}(L)$ for the closed integrable QC (solid curves) and rectangle (dashed curves) billiards. Each data point represents the average over 50000 open trajectories started at random at the boundary. The SO-coupling strength is $\theta_{R} / 2 \pi=0.1$ and 0.3 , while $\theta_{D}=0$.

$\Delta Y=0$, and, therefore, $\mathcal{M}=2$. If the spin is initially polarized in the $X$ direction, the polarization does not change with $L$, i.e., $n_{X}=1$. On the other hand, Eqs. (42) and (45) yield $n_{z}$ $=\mathcal{M} / 2$ if the trajectory starts with $n_{z}=1$. This demonstrates that the spin-relaxation measure $\bar{n}_{z}(L)$ depends on the choice of the quantization axis, as was mentioned in Sec. IV.

\section{Limit of slow spin motion}

Above we presented a number of numerical observations regarding the average spin relaxation in two-dimensional billiards. A further insight into the connection of the spin dynamics to the characteristics of the orbital motion can be gained within the limit of slow spin. In this approximation the period of spin precession is, by definition, much longer than the time scale on which the orbital momentum changes.

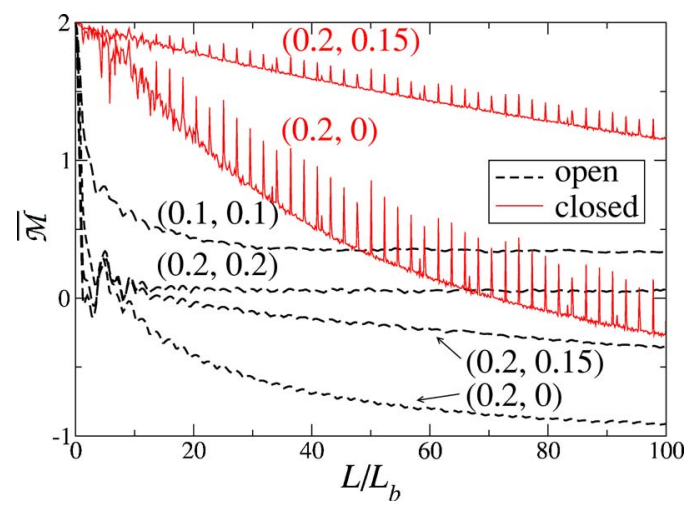

FIG. 10. (Color online) The average modulation factor $\overline{\mathcal{M}}(L)$ for the closed DS billiard at different strengths of the Rashba and Dresselhaus interaction. Each data point represents the average over 50000 open (dashed curves) or 5000 closed (solid curves) trajectories started at random at the boundary. The values of $\left(\theta_{R} / 2 \pi, \theta_{D} / 2 \pi\right)$ are shown on the graph. The leads are parallel to the [100] direction. Note that $\mathcal{M}=2$ for closed orbits, when $\theta_{R}$ $=\theta_{D}$.
In billiards this requirement takes the form $|\mathbf{C}| L_{b} / v \ll 2 \pi$, or, $\theta_{X, Y} \ll 2 \pi$.

\section{Rotation-angle expansion}

The rotation angle $\boldsymbol{\eta}$ [Eq. (53)] contains all the information about the spin evolution along a particular trajectory. It essentially depends on the rotation angles $\boldsymbol{\eta}_{j}$ for the straight segments of the trajectory. In turn, the angles $\boldsymbol{\eta}_{j}$ are directly related to the orbital displacements via Eq. (55). Thus, a more explicit expression of $\boldsymbol{\eta}$ in terms of the $\boldsymbol{\eta}_{j}$ is desirable in order to establish the link between the geometry of orbital motion and the spin rotation. For this purpose we employ the Baker-Campbell-Hausdorff (BCH) formula ${ }^{72,73}$ for the product of the exponentials of two matrices (or operators),

$$
\exp (P) \exp (Q)=\exp \left(\sum_{i=1}^{\infty} R_{i}\right)
$$

where $R_{i}$ 's are homogeneous polynomials of degree $i$ in $P$ and $Q$. The first three of them are given by

$$
\begin{gathered}
R_{1}=P+Q, \\
R_{2}=\frac{1}{2}[P, Q], \\
R_{3}=\frac{1}{12}[P-Q,[P, Q]]
\end{gathered}
$$

([ , ] denotes a commutator). The $\mathrm{BCH}$ formula can be used to calculate the product of the segment propagators $W_{j}$ in Eq. (53). However, only in the limit of slow spin, when $\left|\boldsymbol{\eta}_{j}\right| \ll 1$, the first few contributions $R_{i}$ are sufficient. The rotation angle for a trajectory,

$$
\boldsymbol{\eta}=\boldsymbol{\eta}^{(0)}+\delta \boldsymbol{\eta}^{\perp}+\delta \boldsymbol{\eta}^{\|}
$$

comprises three parts. The lowest-order term,

$$
\boldsymbol{\eta}^{(0)}=\sum_{j=1}^{l} \boldsymbol{\eta}_{j}=2 \pi\left(\frac{\Delta Y}{\Lambda_{X}} \mathbf{e}_{X}+\frac{\Delta X}{\Lambda_{Y}} \mathbf{e}_{Y}\right),
$$

is a vector sum of the segment rotation angles $(\Delta X$ $\left.=\sum_{j=1}^{l} \Delta X_{j}\right)$. The correction normal to the billiard plane,

$$
\delta \boldsymbol{\eta}^{\perp}=\frac{(2 \pi)^{2}}{\Lambda_{X} \Lambda_{Y}} \mathrm{~A} \mathbf{e}_{z}+\mathcal{O}\left(\Lambda_{X, Y}^{-4}\right),
$$

is proportional to the effective enclosed area A (Appendix E). For closed orbits, A coincides with the area defined below Eq. (34) for a returning orbit or a loop. In an open trajectory, A is the area of its "closure" obtained by connecting the endpoints with a straight line. The in-plane correction $\delta \boldsymbol{\eta}^{\|}$is of the order $\Lambda_{X, Y}^{-3}$. In general, the in-plane (normal) contribution to $\boldsymbol{\eta}$ contains odd (even) powers of $\boldsymbol{\eta}_{j}$.

Expressions (63) and (64) help to interpret some of the results of Sec. V B.

(i) The suppression of spin relaxation along open trajectories in confined systems, as compared to extended diffusive systems (Fig. 5), can be deduced from the expansions 


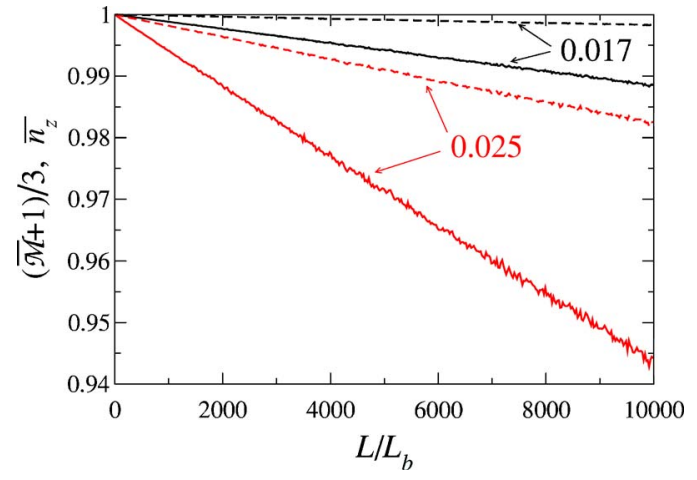

FIG. 11. (Color online) Average spin projection $\overline{n_{z}}(L)$ (dashed curves) and rescaled modulation factor $[\overline{\mathcal{M}}(L)+1] / 3$ (solid curves) for the closed chaotic DS billiard. Each data point represents the average over 15000 closed trajectories started at random at the boundary. The SO-coupling strength $\theta_{R} / 2 \pi$ is shown in the graph and $\theta_{D}=0$.

$$
\left.\begin{array}{c}
\mathcal{M} / 2 \\
n_{z}
\end{array}\right\}=1-\frac{1}{2}\left|\boldsymbol{\eta}^{(0)}\right|^{2}+\mathcal{O}\left(\Lambda_{X, Y}^{-4}\right)
$$

that follow from Eqs. (43) and (45). In billiards $\overline{\left|\boldsymbol{\eta}^{(0)}\right|^{2}}$ increases from zero to its saturation value of the order of $\left(\theta_{X, Y}\right)^{2}$ on the length scale of the system size. The further relaxation on the scale $L \gtrsim L_{b}$ is due to the $\Lambda_{X, Y}^{-4}$-order terms. In diffusive systems, on the other hand, $\overline{\left|\boldsymbol{\eta}^{(0)}\right|^{2}}$ grows linearly with length.

(ii) For the closed orbits one sets $\boldsymbol{\eta}^{(0)}=0$. Hence, the short-scale relaxation does not show up in the ensemble averages $\overline{\mathcal{M}}(L)$ and $\overline{n_{z}}(L)$ (Fig. 6). If the in-plane contribution $\delta \boldsymbol{\eta}^{\|}$is neglected, i.e., $|\delta \boldsymbol{\eta} \|| \ll 1$, the modulation factor becomes

$$
\mathcal{M} \simeq 2 \cos \left|\delta \boldsymbol{\eta}^{\perp}\right|
$$

and the generalized modulation factor is

$$
\mathcal{M} \varphi \simeq \sum_{ \pm} \exp \left[i \mathrm{~A}\left(\frac{4 \pi B_{z}}{\Phi_{0}} \pm \frac{(2 \pi)^{2}}{\Lambda_{X} \Lambda_{Y}}\right)\right]
$$

In a chaotic system, averaging with the area distribution (38) yields

$$
\overline{\mathcal{M} \varphi}\left(L ; B_{z}\right) \simeq \sum_{ \pm} \exp \left(-\left(\widetilde{B} \pm \tilde{\Lambda}^{-2}\right)^{2} \frac{L}{L_{b}}\right)
$$

where $\tilde{\Lambda}^{-2} \equiv 2 \sqrt{2} \pi^{2} A_{0} / \Lambda_{X} \Lambda_{Y}$. Clearly, the normal contribution $\delta \boldsymbol{\eta}^{\perp}$ alone cannot make $\overline{\mathcal{M}} \varphi$ negative. For sufficiently large lengths $L$, the components $\delta \boldsymbol{\eta}^{\perp}$ and $\delta \boldsymbol{\eta}^{\|}$become comparable and reverse the sign of the modulation factor. With the help of Eqs. (42) and (45), we obtain the spin polarization

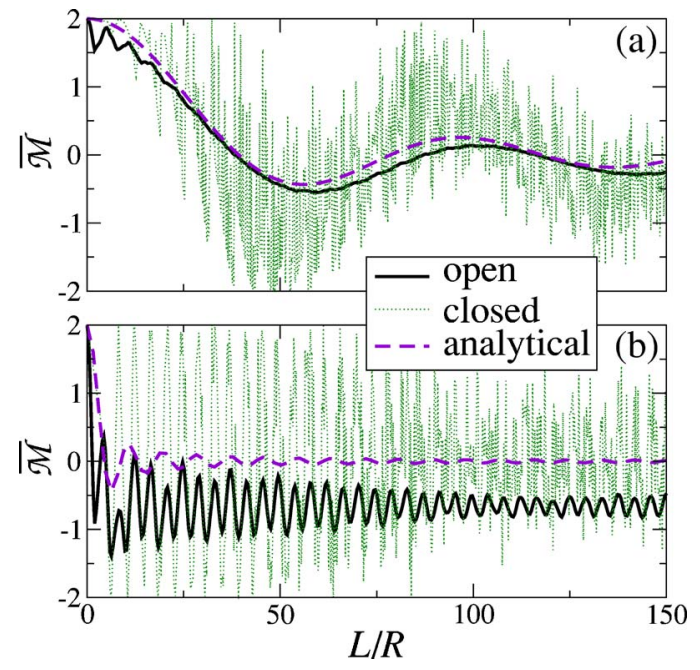

FIG. 12. (Color online) The average modulation factor $\overline{\mathcal{M}}(L)$ for the closed circle billiard of radius $R$. Each data point represents the average over 50000 open (solid curves) or 5000 closed (dotted curves) trajectories started at random at the boundary. The results are compared with the analytical expression (70) (dashed curves). The SO-coupling strength is $\theta_{R} / 2 \pi=\pi R / 2 \Lambda_{R}=0.1$ (a) and 0.3 (b), while $\theta_{D}=0$.

$$
n_{z}=1-2\left(m_{x}^{2}+m_{y}^{2}\right)\left(\sin \frac{\eta}{2}\right)^{2} \simeq 1-2 \frac{\left|\delta \boldsymbol{\eta}^{\|}\right|^{2}}{\left|\delta \boldsymbol{\eta}^{\perp}\right|^{2}}\left(\sin \frac{\left|\delta \boldsymbol{\eta}^{\perp}\right|}{2}\right)^{2} .
$$

The $\delta \boldsymbol{\eta}^{\|}$component is responsible for the rotation of an initially spin-up state. This means that for sufficiently weak SO interaction and short $L, \overline{n_{z}}$ relaxes slower than $\overline{\mathcal{M}}$ (Fig. 11): the relaxation rates are of the order of $\Lambda_{X, Y}^{-6}$ and $\Lambda_{X, Y}^{-4}$, respectively. For stronger interaction the difference is not noticeable (Fig. 6).

(iii) In integrable billiards the strong area cancellation 49 along trajectories contributes to the saturation of spin relaxation. The circle billiard makes an exception, here all the trajectories accumulate area linearly in time. For an orbit having the shortest distance $r$ from the center, the enclosed area is, on average, $A \simeq \pm r L / 2$ (the signs denote the two senses of rotation). Averaging the modulation factor (67) over all $\pm r$, i.e., assuming that all angular momenta are equally possible, we find ${ }^{44}$

$$
\begin{gathered}
\overline{\mathcal{M} \varphi}\left(L ; B_{z}\right) \simeq \sum_{ \pm} \frac{\sin \alpha_{ \pm}}{\alpha_{ \pm}}, \\
\alpha_{ \pm} \equiv 2 \pi R L\left(\frac{B_{z}}{\Phi_{0}} \pm \frac{\pi}{\Lambda_{X} \Lambda_{Y}}\right),
\end{gathered}
$$

where $R$ is the radius of the circle. [Note that a similar average yields $L_{b}=\pi R / 2$, in accordance with Eq. (27).] Surprisingly, for a sufficiently small SO coupling, the average over open trajectories agrees better with Eq. (70), than the average over the closed orbits started at the boundary [Fig. 12(a)]. This happens because the $\boldsymbol{\eta}^{(0)}$ contribution is small, but different angular momenta are not equally represented in the 
ensemble of closed orbits. As well as Eq. (68), Eq. (70) does not describe the full relaxation at large SO interaction [Fig. 12(b)].

\section{Unitary transformation of the Hamiltonian}

It is possible to transform the Rashba-Dresselhaus Hamiltonian [Eqs. (3) and (51)] to the form in which the SO interaction is weaker by a factor of $\theta_{X, Y} \ll 1$ (Refs. 42 and 43). Considering the case $\mathbf{B}=0$, we start with the Hamiltonian

$$
\hat{H}=\frac{\hat{\mathbf{p}}^{2}}{2 M}+\frac{\pi \hbar}{M}\left(\frac{\hat{p}_{Y} \sigma_{X}}{\Lambda_{X}}+\frac{\hat{p}_{X} \sigma_{Y}}{\Lambda_{Y}}\right)
$$

and apply the unitary transformation

$$
\hat{V}=\exp \left[-i \pi\left(\frac{Y \sigma_{X}}{\Lambda_{X}}+\frac{X \sigma_{Y}}{\Lambda_{Y}}\right)\right]
$$

to it. In the limit of slow spin motion, the exponent can be expanded in powers of $\Lambda_{X, Y}^{-1}$. Keeping only the terms quadratic in $\theta_{X, Y}$ and linear in $\hbar / \Lambda_{X, Y}$ [due to the weak-coupling assumption (10)], we obtain the Hamiltonian

$$
\hat{\tilde{H}}=\hat{V}^{\dagger} \hat{H} \hat{V}=\frac{\hat{\mathbf{p}}^{2}}{2 M}-\left(\mathbf{A}^{(1)}+\mathbf{A}^{(2)}\right) \cdot \frac{\hat{\mathbf{p}}}{M}+\mathcal{O}\left[\theta_{X, Y}^{3},\left(\frac{\hbar}{\Lambda_{X, Y}}\right)^{2}\right] .
$$

It contains the spin-dependent "vector potentials,"

$$
\begin{gathered}
\mathbf{A}^{(1)}=-\frac{\pi^{2} \hbar}{\Lambda_{X} \Lambda_{Y}}\left(\mathbf{e}_{z} \times \mathbf{r}\right) \sigma_{z}, \\
\mathbf{A}^{(2)}=\frac{4 \pi^{3} \hbar}{3 \Lambda_{X} \Lambda_{Y}}\left(\mathbf{e}_{z} \times \mathbf{r}\right)\left(\frac{X \sigma_{X}}{\Lambda_{Y}}-\frac{Y \sigma_{Y}}{\Lambda_{X}}\right)
\end{gathered}
$$

of the order of $\left(\hbar / \Lambda_{X, Y}\right) \theta_{X, Y}$ and $\left(\hbar / \Lambda_{X, Y}\right) \theta_{X, Y}^{2}$, respectively.

As any two Hamiltonians connected by a unitary transformation, $\hat{H}$ and $\hat{\tilde{H}}$ must yield the same total transmission $\mathcal{T}$ and reflection $\mathcal{R}$. While $\hat{H}$ and $\hat{\widetilde{H}}$ have identical orbital parts, the SO interaction in the latter is much weaker. It is important, therefore, to understand the role of the transformation $\hat{V}$ within the framework of the semiclassical Landauer formula developed in the preceding sections. Working in the weakcoupling regime (10) and treating the orbital motion semiclassically, we consider the unitary transformation

$$
V(t)=\exp \left[-i \pi\left(\frac{Y(t) \sigma_{X}}{\Lambda_{X}}+\frac{X(t) \sigma_{Y}}{\Lambda_{Y}}\right)\right]
$$

acting in the spin space. It is generated by a classical trajectory $[\mathbf{r}(t), \mathbf{v}(t)]$. The spin-propagator matrix (41) transforms as

$$
\tilde{W}(t)=V^{\dagger}(t) W(t) V(0) .
$$

[This property follows from the spinor transformation $\widetilde{\psi}(t)$ $=V^{\dagger}(t) \psi(t)$ and the definition $\tilde{\psi}(t)=\tilde{W}(t) \tilde{\psi}(0)$.] For a closed orbit of time $t$, we have $V(t)=V(0)$. Hence, the modulation factor for a pair of trajectories from the diagonal or the loop contribution (Sec. III),

$$
\tilde{\mathcal{M}}(t) \equiv \operatorname{Tr}[\tilde{W}(t)]^{2}=\mathcal{M}(t),
$$

is preserved under the transformation. Thus, $\hat{H}$ and $\hat{\widetilde{H}}$ return the same conductance also in the semiclassical approximation. ${ }^{92}$ This result demonstrates the significance of closed trajectories for the transmission and reflection.

According to Eq. (78), the modulation factor for closed orbits can be determined directly from the semiclassical version of $\hat{\widetilde{H}}$. Neglecting $\mathbf{A}^{(2)}$, we compute the propagator as

$$
\tilde{W}(t) \simeq \exp \left(\frac{i}{\hbar} \int_{0}^{t} \mathbf{A}^{(1)} \cdot \mathbf{v} d t^{\prime}\right)=\exp \left(-i \frac{2 \pi^{2}}{\Lambda_{X} \Lambda_{Y}} \mathrm{~A} \sigma_{z}\right) .
$$

Then Eq. (64) for the rotation angle and Eq. (67) for the modulation factor (with $\mathbf{B}=0$ ) follow.

For the open trajectories, the leading-order (in $\Lambda_{X, Y}^{-1}$ ) contribution from Eq. (77) is

$$
\begin{aligned}
W(t) & \simeq V(t) V^{\dagger}(0) \\
& \simeq \exp \left[-i \pi\left(\frac{Y(t)-Y(0)}{\Lambda_{X}} \sigma_{X}+\frac{X(t)-X(0)}{\Lambda_{Y}} \sigma_{Y}\right)\right] .
\end{aligned}
$$

As a consequence, Eqs. (63) and (65) are obtained.

\section{RASHBA AND DRESSELHAUS INTERACTION: QUANTUM CORRECTIONS TO TRANSMISSION AND REFLECTION}

\section{A. Results of the semiclassical theory}

As was shown in the preceding sections, the average relaxation of the spin modulation factor as a function of orbit length is closely related to the type of the classical dynamics of the system. In view of Eq. (37), the effect of the classical orbital motion is also felt in the magnitude and sign of the quantum correction to the conductance.

In the examples below the following numerical procedure is employed to calculate the relative quantum corrections: The trajectories are started at the boundary within a given lead. Their initial coordinate and velocity component at the lead cross section is chosen at random. If an orbit returns to the same lead, the generalized modulation factor (36) for this orbit and its time-reversed partner is recorded. The average over these modulation factors yields the diagonal contribution $^{93} \delta \mathcal{R}_{\text {diag }}(\mathbf{B}) / \delta \mathcal{R}_{\text {diag }}^{(0)}$, which, by virtue of $\mathrm{Eq}$. (37), is equal to the total relative quantum corrections $\delta \mathcal{R}(\mathbf{B}) / \delta \mathcal{R}^{(0)}=\delta \mathcal{T}(\mathbf{B}) / \delta \mathcal{T}^{(0)}$ for a chaotic system. For nonchaotic billiards there exist no analytical approach to the loop statistics. Hence, there we use the diagonal part as an estimate for $\delta \mathcal{R}(\mathbf{B}) / \delta \mathcal{R}^{(0)}$.

In Fig. 13 the relative quantum correction is shown as a function of the Rashba-interaction strength $\theta_{R}$ (while $\theta_{D}=0$ ) for several billiards. Here and in the following figures the results for lead "1" (Fig. 4) are presented. All the billiards have the same ratio $\mathrm{P}_{c} /\left(w+w^{\prime}\right)$, which in chaotic systems gives the escape length in units of $L_{b}$. As $\theta_{R}$ increases, the 


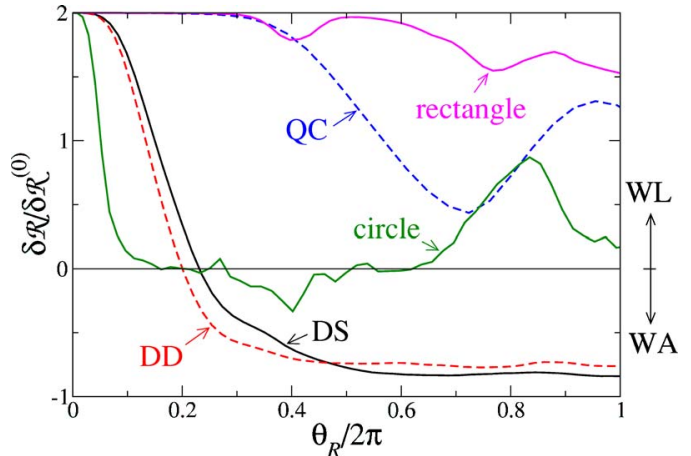

FIG. 13. (Color online) Relative quantum correction to reflection (or transmission) coefficients vs. Rashba-interaction strength $\theta_{R}\left(\theta_{D}=0\right)$. The data are shown for the chaotic (DS and DD) and integrable (QC, rectangle, and circle) billiards with $\mathrm{P}_{c} /\left(w+w^{\prime}\right)$ =90. Each data point represents the average over 50000 orbits. The details on the numerical procedure are given in the text.

relative quantum corrections in chaotic cavities decrease and, eventually, change sign. The level $\delta \mathcal{R} / \delta \mathcal{R}^{(0)}=0$ corresponds to the WL-WA transition. In the integrable QC and rectangle billiards no WA occurs, while the circle billiard shows a non-typical pattern.

The interplay between Rashba and Dresselhaus interaction in a chaotic billiard is investigated in Fig. 14. As was already noted in Sec. V B, the two interactions tend to compensate each other (thus suppressing the WA) as their ratio gets closer to unity. When $\theta_{R}=\theta_{D}$, there is no manifestation of SO interaction in the conductance $\left[\delta \mathcal{R}(\mathbf{B}=0) / \delta \mathcal{R}^{(0)} \simeq 2\right]$, since, effectively, no spin relaxation is associated with closed trajectories.

Owing to the scaling property mentioned below Eq. (55), the quantum corrections for a given billiard shape depend only on $\theta_{R}$ and $\theta_{D}$. These parameters are proportional to the size of the system. Thus, in a material with definite physical SO-coupling constants $\alpha_{R}$ and $\alpha_{D}$, Fig. 14 (curves with constant $\left.\theta_{R} / \theta_{D}\right)$ predicts the suppression of WA with the decreasing size of a chaotic quantum dot (also observed in experiments ${ }^{32}$ ). Preserving the shape of the cavity means, in

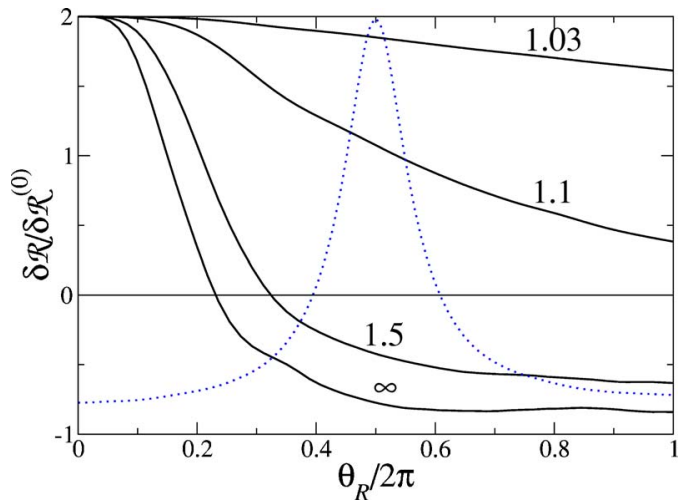

FIG. 14. (Color online) The relative quantum correction to reflection (or transmission) coefficients as a function of $\theta_{R}$ for the chaotic DS billiard with $\mathrm{P}_{c} /\left(w+w^{\prime}\right)=90$. Solid curves: fixed ratios $\theta_{R} / \theta_{D}$ (shown in the graph). Dotted curve: fixed $\theta_{D}=\pi$. Each data point represents the average over 50000 orbits.

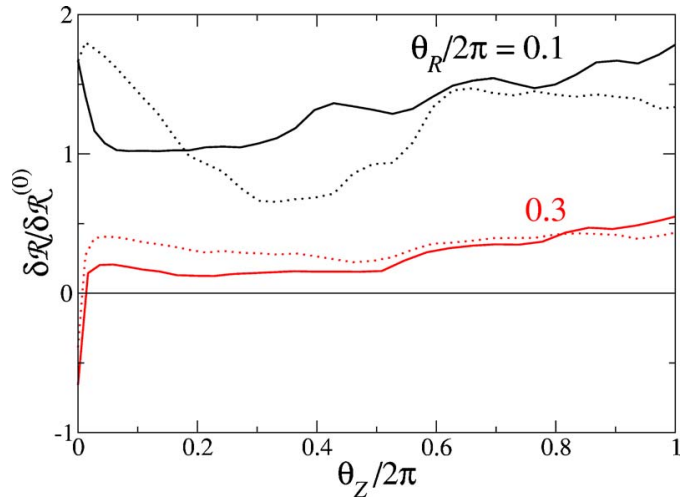

FIG. 15. (Color online) The relative quantum correction to reflection (or transmission) coefficients as a function of the Zeemancoupling strength $\theta_{Z}$ for the chaotic DS billiard with $\mathrm{P}_{c} /\left(w+w^{\prime}\right)$ $=90$. The in-plane magnetic field is parallel (solid curves) or perpendicular (dotted curves) to the leads' direction (see Fig. 4). Two Rashba-coupling strengths $\theta_{R} / 2 \pi=0.1,0.3$ (shown in the graph) are considered. The Dresselhaus interaction is absent. Each data point represents the average over 50000 orbits.

particular, that $\mathrm{P}_{c} /\left(w+w^{\prime}\right)$ is kept constant when the dot size is changed. Alternatively, one can fix $w+w^{\prime}$ : this leads to a disproportionate decrease of $L_{\text {esc }}$ when the size is reduced, thereby suppressing WA even stronger.

In experiments, for fixed $\theta_{D}, \theta_{R}$ can be tuned through an additional back-gate potential. The dotted curve in Fig. 14 shows the result of such a parameter variation, including the associated WA-WL-WA crossover.

The effect of Zeeman interaction in the chaotic DS billiard is considered in Fig. 15. The interaction strength is measured by the spin-precession angle per bounce $\theta_{Z}$ $=L_{b}\left|\mathbf{C}_{Z}\right| / v$, where $\mathbf{C}_{Z}$ is the Zeeman contribution to the effective field $\mathbf{C}$. We observe a complete suppression of WA even by weak Zeeman coupling. In general, the $\theta_{Z}$ dependence of $\delta \mathcal{R} / \delta \mathcal{R}^{(0)}$ is very complex. It shows strong anisotropy with respect to the direction of the applied in-plane magnetic field, especially, for weak SO interaction. Note that the symmetry of the spin relaxation under the $\Lambda_{R} \leftrightarrow \Lambda_{D}$ exchange (Sec. V A) is lifted, unless $\mathbf{C}_{Z}$ lies in the (110) plane.

\section{B. Comparison with full quantum mechanics}

The semiclassical results are compared with quantum calculations in Figs. 16 and 17. The complete quantum transmission and reflection amplitudes $t_{n \sigma^{\prime}, m \sigma}$ and $r_{n \sigma^{\prime}, m \sigma}$ are computed numerically by using a recursive Green-function technique (see Ref. 74 and references therein for details). This approach is based on a tight-binding model arising from the real-space discretization of the spin-dependent Schrödinger equation in a two-dimensional geometry. Here, the standard on-site and hopping energies present in a spinless tight-binding model are replaced by $2 \times 2$ matrices accounting for the spin degree of freedom. ${ }^{94}$ In combination with a recursive algorithm, we obtain the Green function describing spin-dependent transport between the leads by solving the (implicit) Dyson equation. The amplitudes $t_{n \sigma^{\prime}, m \sigma}$ and $r_{n \sigma^{\prime}, m \sigma}$ are evaluated by projecting the Green function 


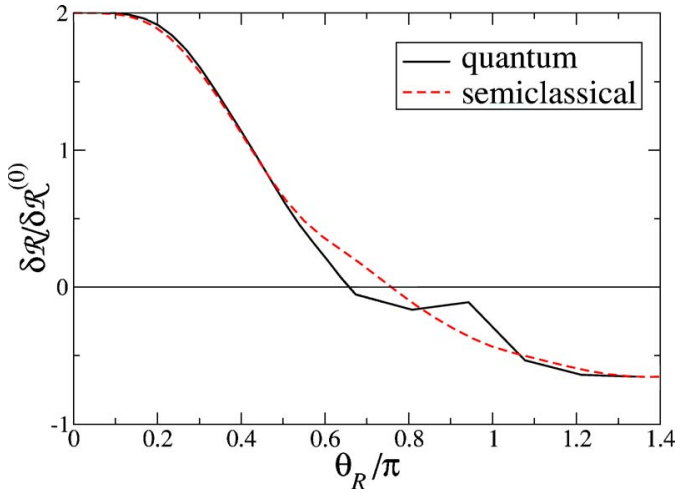

FIG. 16. (Color online) Relative quantum correction to reflection coefficient vs Rashba-interaction strength $\theta_{R}\left(\theta_{D}=0\right)$ in the DS billiard with $\mathrm{P}_{c} /\left(w+w^{\prime}\right)=30$. The quantum reflection was computed for the energy corresponding to $N=N^{\prime}=6$ open channels in the leads. The relative quantum correction (solid curve) was obtained after estimating the classical reflection $\mathcal{R}_{\mathrm{cl}} \simeq 6.49$ as described in the text. In the semiclassical case (dashed curve) each data point represents the average over 50000 orbits.

onto a complete set of asymptotic states defining the incoming and/or outgoing spin and orbital channels in the leads. The method has the advantage of large flexibility, the quantum amplitudes can be equally calculated for geometries of the arbitrary shape. Moreover, disorder, magnetic fields, and spin-dependent interactions can be easily introduced and modified.

The dependence of $\delta \mathcal{R} / \delta \mathcal{R}^{(0)}$ on the Rashba-coupling strength in the DS billiard is presented in Fig. 16. Compared to Fig. 13, here we choose a smaller ratio $\mathrm{P}_{c} /\left(w+w^{\prime}\right)$ in order to reduce the computing time in the quantum case. There is a good overall agreement between the two curves, especially, above the WL-WA transitional region. Since full quantum calculations give only the total reflection, one is faced with the problem of separating the quantum correc-

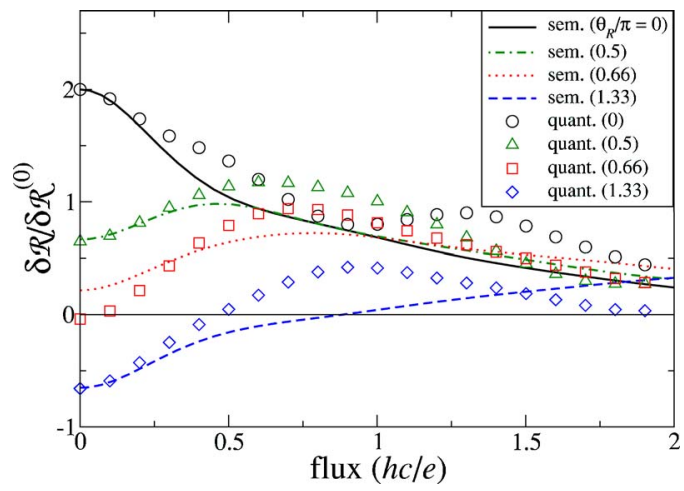

FIG. 17. (Color online) The relative quantum correction to reflection coefficient vs magnetic flux in the DS billiard with $\mathrm{P}_{c} /(w$ $\left.+w^{\prime}\right)=30$. The quantum reflection was computed for the energy corresponding to $N=N^{\prime}=6$ open channels in the leads. In the semiclassical case each data point represents the average over 50000 orbits. The Rashba interaction was kept constant at $\theta_{R} / \pi=0$ (solid curve, semiclassical; circles, quantum); 0.5 (dashed-dotted curve, triangles); 0.66 (dotted curve, squares); 1.33 (dashed curve, diamonds). The Dresselhaus and Zeeman couplings were not included. tions from the classical part. Instead of evaluating $\mathcal{R}_{\mathrm{cl}}$ directly, we estimate it by requiring that the ratio $\delta \mathcal{R}\left(\theta_{R}\right.$ $=0) / \delta \mathcal{R}\left(\theta_{R} \gg 1\right)$ is the same in the semiclassical and quantum consideration.

We used the estimated value of $\mathcal{R}_{\mathrm{cl}}$ for calculating the quantum corrections in the presence of magnetic flux $B_{z} A_{c} / \Phi_{0}$ (Fig. 17). In this graph the quantum results exhibit additional oscillations around the semiclassical curves, both with and without the SO interaction. The observed deviations from the semiclassics might be due to the fact that, for the energy range considered in the quantum calculations, the bending of classical trajectories caused by a Lorentz force limits the applicability of the theory in Sec. III C. By the same token, the change of the trajectories resulting from the SO interaction might be responsible for the discrepancy in Fig. 16. Nevertheless, the semiclassical techniques prove to be an effective tool for making reasonable predictions with a minimum of computational power. ${ }^{95}$

The maximum in $\delta \mathcal{R}\left(B_{z}\right) / \delta \mathcal{R}^{(0)}$, clearly noticeable at the intermediate SO interaction (Fig. 17), can be attributed to the cancellation between the external flux and the flux of the SO vector potential (74). ${ }^{43}$ Equivalently, the integration (37) with the modulation factor (68) yields [cf. Eq. (39)]

$$
\frac{\delta \mathcal{R}(\mathbf{B})}{\delta \mathcal{R}^{(0)}} \simeq \sum_{ \pm} \frac{1}{1+\left(\widetilde{B} \pm \tilde{\Lambda}^{-2}\right)^{2} L_{\mathrm{esc}} / L_{b}} .
$$

Thus, the maxima are located at $\widetilde{B} \approx \pm \widetilde{\Lambda}^{-2}$. Note that the above expression, being positive, does not describe the WA, which appears due to the $\delta \boldsymbol{\eta}$ spin rotation (see Sec. V C).

\section{SUMMARY AND CONCLUSIONS}

The semiclassical Landauer formula with spin expresses quantum corrections to transmission and reflection in ballistic quantum dots as a sum over pairs of classical trajectories or their loop parts, related by time reversal. The effect of the spin-orbit and Zeeman interaction, as well as the AharonovBohm phase, can be taken into account by averaging of a modulation factor over the ensemble of closed orbits. The change of classical trajectories by the spin-orbit interaction and the Lorentz force can be neglected, provided the energy of the particle is sufficiently high. With the increasing spinorbit coupling strength the quantum corrections may reverse their signs, and weak localization will become weak antilocalization. Whether such a transition takes place, depends on the interplay between the average spin relaxation and the dwell time in the quantum dot.

The spin relaxation, particularly for Rashba and Dresselhaus interaction, is very sensitive to the character of classical dynamics, which is determined by the shape of the boundary. While in chaotic geometries spin eventually completely relaxes, in integrable systems (except for the circle billiard) the relaxation is strongly suppressed. Consequently, at a given spin-orbit coupling strength, a chaotic cavity can be in the weak-antilocalization regime, whereas in a corresponding integrable cavity weak localization will take place. Weak antilocalization is suppressed by an external magnetic field via the Zeeman interaction and the Aharonov-Bohm phase. The 
size reduction of the quantum dot works against the spin relaxation and the antilocalization, as well. Rashba and Dresselhaus interactions compensate the effects of each other on spin relaxation and transport.

The degree of spin relaxation for closed orbits, which controls the quantum corrections to transport coefficients, is reduced, compared to open trajectories of the same length. This brings about an important difference in the semiclassical treatment of systems with and without spin-orbit coupling, in the latter case, the modulation factor due to magnetic flux can be averaged over arbitrary trajectories. In the limit of slow spin dynamics, the restriction to closed orbits is lifted, if the leading-order Rashba and Dresselhaus terms are removed from the Hamiltonian by a gauge transformation.

The semiclassical results for a chaotic billiard with moderate spin-orbit interaction and weak magnetic field show a good agreement with the quantum calculations. To account for the deviations at larger values, it may be necessary to include the distortion of classical trajectories in the semiclassical analysis. Possible extensions of the theory could be based on the extended-phase-space approach to spin-orbit coupling. ${ }^{54,56}$

\section{ACKNOWLEDGMENTS}

The authors thank L. E. Golub for helpful discussions. The work was supported by the Deutsche Forschungsgemeinschaft through the Research group FOR 370 and the Research school GRK 638 (O.Z. and K.R.) and by the EU Spintronics Research Training Network (D.F.).

\section{APPENDIX A: SPIN PROPAGATOR}

Let us consider a spin, precessing in a time-dependent magnetic field $\mathbf{C}(t)$, with the Hamiltonian $\hat{H}(t)=\hbar \hat{\mathbf{s}} \cdot \mathbf{C}(t)$. The path integral (9) for the corresponding propagator $K\left(\zeta_{2}, \zeta_{1} ; t\right)$ in the coherent-state representation yields ${ }^{60}$

$$
K\left(\zeta_{2}, \zeta_{1} ; t\right)=\frac{\left[a^{*}(t)-b^{*}(t) \zeta_{1}+b(t) \zeta_{2}^{*}+a(t) \zeta_{2}^{*} \zeta_{1}\right]^{2 s}}{\left(1+\left|\zeta_{2}\right|^{2}\right)^{s}\left(1+\left|\zeta_{1}\right|^{2}\right)^{s}}
$$

where the coefficients $a(t)$ and $b(t)$ are found from the differential equation,

$$
\frac{d W(t)}{d t}=-\frac{i}{2} \boldsymbol{\sigma} \cdot \mathbf{C}(t) W(t), \quad W(0)=1
$$

for the matrix

$$
W(t) \equiv\left(\begin{array}{cc}
a(t) & b(t) \\
-b^{*}(t) & a^{*}(t)
\end{array}\right) \in \mathrm{SU}(2) .
$$

In Eq. (A2) $\boldsymbol{\sigma}$ is the vector of Pauli matrices. As any $\mathrm{SU}(2)$ matrix, $W(t)$ can be represented in the form

$$
W(t)=1 \cos \frac{\eta(t)}{2}-i \boldsymbol{\sigma} \cdot \mathbf{m}(t) \sin \frac{\eta(t)}{2}=e^{-i \boldsymbol{\sigma} \cdot \boldsymbol{\eta}(t) / 2},
$$

where $\mathbf{m}(t) \equiv \boldsymbol{\eta}(t) / \eta(t)$ is a unit vector. The spin $s$ timeevolution operator (propagator) $\hat{K}(t)$ that belongs to the $(2 s$ +1)-dimensional irreducible representation of $\mathrm{SU}(2)$, satisfies the equation

$$
\frac{d \hat{K}}{d t}=-\frac{i}{\hbar} \hat{H}(t) \hat{K}=-i \hat{\mathbf{s}} \cdot \mathbf{C}(t) \hat{K}, \quad \hat{K}(0)=\hat{1},
$$

yielding

$$
\hat{K}(t)=e^{-i \hat{\mathbf{s}} \cdot \boldsymbol{\eta}(t)}
$$

Thus, $\hat{K}(t)$, applied to the spin states at time $t=0$, rotates them by the angle $\eta(t)$ about the axis $\mathbf{m}(t)$. The coherentstate propagator (A1) is the matrix element of $\hat{K}(t)$,

$$
K\left(\zeta_{2}, \zeta_{1} ; t\right)=\left\langle\zeta_{2}|\hat{K}(t)| \zeta_{1}\right\rangle=\left\langle\zeta_{2}\left|e^{-i \hat{s} \cdot \boldsymbol{\eta}(t)}\right| \zeta_{1}\right\rangle .
$$

Note that, setting $t=0$ (i.e., $a=1, b=0$ ) in Eq. (A1), we obtain an explicit expression for the scalar product $\left\langle\zeta_{2} \mid \zeta_{1}\right\rangle$ of two coherent states.

\section{APPENDIX B: LOOP CONTRIBUTION}

In Ref. 50 the loop correction was derived for a hyperbolic system with a single Lyapunov exponent $\lambda$. Here we extend this derivation to the case with SO interaction, or, indeed, any interaction that does not change the classical trajectories in the leading semiclassical order.

The action difference $\Delta \mathcal{S}\left(\varepsilon_{\gamma, \gamma^{\prime}}\right) \simeq M v^{2} \varepsilon_{\gamma, \gamma^{\prime}}^{2} / 2 \lambda$ for a loop pair of trajectories $\gamma$ and $\gamma^{\prime}$ (in the absence of magnetic field) depends on the crossing angle $\varepsilon_{\gamma, \gamma^{\prime}}$, as well as the velocity $v$ and the mass $M$. Any long orbit has many selfcrossings, each corresponding to a loop. For an orbit of length $L$, the average number of loops of length between $l$ and $l+d l$ and crossing angle between $\varepsilon$ and $\varepsilon+d \varepsilon$ is

$$
P_{\text {loop }}(l, \varepsilon ; L) d l d \varepsilon \simeq \frac{(L-l) \sin \varepsilon}{\pi \mathrm{A}_{c}} d l d \varepsilon .
$$

The loop length lies in the interval $l_{\text {min }}(\varepsilon)<l<L$, where $l_{\min }(\varepsilon) \sim-(2 v / \lambda) \ln (\varepsilon /$ const $)$ is the minimal possible loop length. This length is of order of the size of the crossing region where the intersecting segments of the trajectory are interrelated by the linearized dynamics and, therefore, cannot form a closed loop.

The loop correction to the channel-resolved transmission coefficient $\left|t_{n m}\right|_{\text {loop }}^{2}$ can be expressed as a sum over all trajectories $\gamma(\bar{n}, \bar{m})$ weighted with the number of loops (B1) per orbit:

$$
\begin{aligned}
\left|t_{n m}\right|_{\text {loop }}^{2}= & 2 \sum_{\substack{\text { loop } \\
\text { pairs }}} \mathcal{A}_{\gamma} \mathcal{A}_{\gamma^{\prime}}^{*} \cos \left(\frac{\Delta \mathcal{S}\left(\varepsilon_{\gamma, \gamma^{\prime}}\right)}{\hbar}\right)(\mathcal{M} \varphi)_{\gamma, \gamma^{\prime}} \\
\simeq & 2 \sum_{\gamma(\bar{n}, \bar{m})}\left|\mathcal{A}_{\gamma}\right|^{2} \int_{0}^{\pi} d \varepsilon \int_{l_{\min }(\varepsilon)}^{L_{\gamma}} d l P_{\text {loop }}\left(l, \varepsilon ; L_{\gamma}\right) \\
& \times \cos \left(\frac{\Delta \mathcal{S}(\varepsilon)}{\hbar}\right) \overline{\mathcal{M} \varphi}\left[l-l_{\min }(\varepsilon)\right] .
\end{aligned}
$$

By $\overline{\mathcal{M} \varphi}\left[l-l_{\min }(\varepsilon)\right]$ we denote the average of $(\mathcal{M} \varphi)_{\gamma, \gamma^{\prime}}$ over 
all loops with the effective length $l_{e}=l-l_{\min }(\varepsilon)$. The contribution of the crossing region to $\overline{\mathcal{M} \varphi}$ can be neglected, since, in the semiclassical limit $\Delta \mathcal{S}(\varepsilon) / \hbar \gg 1$, only small $\varepsilon$ are important in the integral (B2). Moreover, only the remaining part of the loop of length $l_{e}$ obeys the statistical assumptions under which the average $\overline{\mathcal{M} \varphi}$ is obtained. Substituting $P_{\text {loop }}\left(l, \varepsilon ; L_{\gamma}\right)$ from Eq. (B1), we compute the $l$ integral

$$
\begin{gathered}
\int_{l_{\min }(\varepsilon)}^{L_{\gamma}} d l P_{\text {loop }}\left(l, \varepsilon ; L_{\gamma}\right) \overline{\mathcal{M} \varphi}\left[l-l_{\min }(\varepsilon)\right] \\
\approx \frac{\sin \varepsilon}{\pi \mathrm{A}_{c}}\left(\int_{0}^{L_{\gamma}} d l_{e}\left(L_{\gamma}-l_{e}\right) \overline{\mathcal{M} \varphi}\left(l_{e}\right)\right. \\
\left.\quad-l_{\min }(\varepsilon) \int_{0}^{L_{\gamma}} d l_{e} \overline{\mathcal{M} \varphi}\left(l_{e}\right)\right)
\end{gathered}
$$

ignoring the terms of order $\left[l_{\min }(\varepsilon)\right]^{2}$. When integrating over $\varepsilon$ in Eq. (B2), we can approximate $\sin \varepsilon \approx \varepsilon$ and extend the integration to infinity. Only the term proportional to $l_{\min }(\varepsilon)$ in Eq. (B3) survives the integration, and we obtain ${ }^{68}$

$$
\int_{0}^{\pi} d \varepsilon \cos \left(\frac{\Delta \mathcal{S}(\varepsilon)}{\hbar}\right) \frac{\sin \varepsilon}{\pi \mathrm{A}_{c}} l_{\min }(\varepsilon) \simeq\left[2\left(N+N^{\prime}\right) L_{\mathrm{esc}}\right]^{-1} .
$$

Using the results of Eqs. (B3) and (B4) in Eq. (B2) and applying the sum rule (24), we factorize the transmission coefficient into a spin- and field-independent part and a length-averaged modulation factor $\langle\overline{\mathcal{M} \varphi}(\mathbf{B})\rangle_{L}$,

$$
\begin{aligned}
\left|t_{n m}\right|_{\text {loop }}^{2} & \simeq-\left(N+N^{\prime}\right)^{-2} L_{\text {esc }}^{-1} \int_{0}^{\infty} d L P_{L}(L) \int_{0}^{L} d l_{e} \overline{\mathcal{M} \varphi}\left(l_{e}\right) \\
& =-\left(N+N^{\prime}\right)^{-2} \int_{0}^{\infty} d l_{e} P_{L}\left(l_{e}\right) \overline{\mathcal{M} \varphi}\left(l_{e}\right) .
\end{aligned}
$$

After the summation over the channels we obtain the loop corrections (37).

\section{APPENDIX C: MODULATION FACTOR FOR AN ARBITRARY SPIN}

To calculate the spin $s$ modulation factor for a diagonal or loop pair of trajectories, we start from Eqs. (32) and (33). Writing $\hat{K}$ in the exponential form (A6), one finds

$$
\mathcal{M}=\operatorname{Tr}\left(\hat{K}^{2}\right)=\operatorname{Tr}\left(e^{-2 i \hat{s} \cdot \boldsymbol{\eta}}\right)=\sum_{\sigma=-s}^{s} e^{-2 i \eta \sigma} .
$$

Here, the last identity was obtained by choosing the $z$ axis in the direction of $\boldsymbol{\eta}$ and using the standard matrix form of $\hat{s}_{z}$. It is sometimes convenient to represent the result in terms of the Gegenbauer polynomials ${ }^{75} C_{j}^{1}$ on the sphere $S^{3}$, which generalize the Legendre polynomials on $S^{2}$. For this purpose we transform

$$
\begin{aligned}
\sum_{\sigma=-s}^{s} e^{-i 2 \eta \sigma} & =\sum_{j=0}^{2 s}(-1)^{j} \sum_{\sigma=-(2 s-j)}^{2 s-j} e^{-i \eta \sigma} \\
& =\sum_{j=0}^{2 s}(-1)^{2 s-j} \frac{\sin [(2 j+1) \eta / 2]}{\sin (\eta / 2)}
\end{aligned}
$$

and apply the property ${ }^{75}$

$$
\frac{\sin [(2 j+1) \eta / 2]}{\sin (\eta / 2)}=C_{2 j}^{1}\left(\cos \frac{\eta}{2}\right) .
$$

Eventually, we arrive at the expansion

$$
\mathcal{M}=\sum_{j=0}^{2 s}(-1)^{2 s-j} C_{2 j}^{1}\left(\xi_{4}\right)
$$

with $\xi_{4}=\cos (\eta / 2)$ [see Eq. (42)].

In the asymptotic limit $L \rightarrow \infty$ of long trajectories, when the spin state is completely randomized, $\overline{\mathcal{M}}(\infty)$ can be determined by averaging of Eq. (C4) over $S^{3}$, which amounts to computing the scalar product of $\mathcal{M}\left(\xi_{4}\right)$ and $C_{0}^{1}\left(\xi_{4}\right)=1$ on the sphere. Then the orthogonality condition for the Gegenbauer polynomials, ${ }^{75}$

$$
\frac{1}{2 \pi^{2}} \int_{S^{3}} d \xi C_{j}^{1}\left(\xi_{4}\right) C_{j^{\prime}}^{1}\left(\xi_{4}\right)=\delta_{j j^{\prime}},
$$

where $d \boldsymbol{\xi}$ is the surface element and $2 \pi^{2}$ is the surface "area," yields $\overline{\mathcal{M}}(\infty)=(-1)^{2 s}$.

\section{APPENDIX D: SPIN DIFFUSION}

The spin evolution in a random magnetic field is mapped onto a random walk on the sphere $S^{3}$ with initial point $\mathbf{e}_{4}$ $\equiv(0,0,0,1)$. In the short-step limit $|\mathbf{C}| \tau / 2 \ll \pi / 2$, the probability density $P_{\text {diff }}(\boldsymbol{\xi} ; t)$ to find the particle at point $\boldsymbol{\xi}$ at time $t$ solves the diffusion equation ${ }^{76}$

$$
\left(\frac{\partial}{\partial t}-D \nabla^{2}\right) P_{\text {diff }}(\boldsymbol{\xi} ; t)=0, \quad P_{\text {diff }}(\boldsymbol{\xi} ; 0)=\delta\left(\boldsymbol{\xi}, \mathbf{e}_{4}\right),
$$

with the diffusion coefficient $D=|\mathbf{C}|^{2} \tau / 24$. Here $\nabla^{2}$ is the Laplacian on $S^{3}$, and $\delta\left(\boldsymbol{\xi}, \mathbf{e}_{4}\right)$ is the $\delta$-function on $S^{3}$ between two points. The probability is normalized by $\int_{S^{3}} d \boldsymbol{\xi} P_{\text {diff }}(\boldsymbol{\xi} ; t)=1$, where $d \boldsymbol{\xi}$ is the surface element. The solution can be found in the form of an expansion in the Gegenbauer polynomials ${ }^{75} C_{j}^{1}$ :

$$
P_{\text {diff }}(\boldsymbol{\xi} ; t)=\frac{1}{2 \pi^{2}} \sum_{j=0}^{\infty}(j+1) e^{-j(j+2) D t} C_{j}^{1}\left(\xi_{4}\right) .
$$

In order to determine the average modulation factor $\overline{\mathcal{M}}_{\text {diff }}(t)$, we express $\mathcal{M}\left(\xi_{4}\right)=C_{2}^{1}\left(\xi_{4}\right)-C_{0}^{1}\left(\xi_{4}\right)$ from Eq. (43). Taking into account the orthogonality condition (C5), we compute the average for the distribution $P_{\text {diff }}(\xi ; t)$,

$$
\overline{\mathcal{M}}_{\text {diff }}(t)=\int_{S^{3}} d \boldsymbol{\xi} P_{\text {diff }}(\boldsymbol{\xi} ; t) \mathcal{M}\left(\xi_{4}\right)=3 e^{-8 D t}-1,
$$

yielding Eq. (46). This result can be generalized to an arbitrary spin $s$ if one employs the representation $(\mathrm{C} 4)$, 


$$
\overline{\mathcal{M}}_{\mathrm{diff}}(t)=\sum_{j=0}^{2 s}(-1)^{2 s-j}(2 j+1) e^{-4 j(j+1) D t} .
$$

The average spin polarization is, according to Eq. (45),

$$
{\overline{\left(n_{z}\right)}}_{\mathrm{diff}}(t)=2\left[\overline{\xi_{3}^{2}}(t)+\overline{\xi_{4}^{2}}(t)\right]-1 .
$$

Using Eqs. (43) and (46), we find

$$
\overline{\xi_{4}^{2}}(t)=\left(3 e^{-(1 / 3)|\mathbf{C}|^{2} \pi t}+1\right) / 4 .
$$

The symmetry of the problem provides $3 \overline{\xi_{3}^{2}}(t)=1-\overline{\xi_{4}^{2}}(t)$, and Eq. (47) follows.

\section{APPENDIX E: AREA CONTRIBUTION TO THE SPIN-ROTATION ANGLE}

To derive Eq. (64), it is convenient to use the notation $\boldsymbol{\eta}_{l, 1} \equiv \boldsymbol{\eta}$ in Eq. (53). When $l=2$, the BCH formula (58) applied to the product of the two segment propagators yields

$$
\boldsymbol{\eta}_{2,1}=\boldsymbol{\eta}_{1}+\boldsymbol{\eta}_{2}-\frac{1}{2} \boldsymbol{\eta}_{1} \times \boldsymbol{\eta}_{2}+\mathcal{O}\left(\Lambda_{X, Y}^{-3}\right) .
$$

For an arbitrary $l$, this result generalizes to

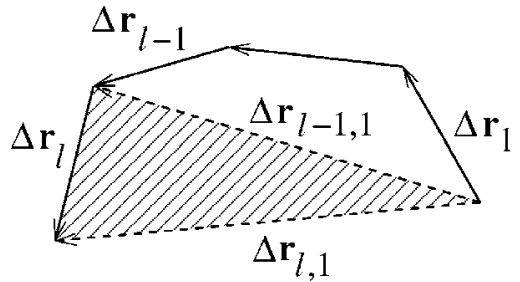

FIG. 18. Definition of the enclosed area [Eq. (E4)]. The shaded area is $(1 / 2) \Delta \mathbf{r}_{l-1,1} \times \Delta \mathbf{r}_{l}$.

$$
\boldsymbol{\eta}_{l, 1}=\boldsymbol{\eta}_{l, 1}^{(0)}-\frac{1}{2} \sum_{j=2}^{l} \boldsymbol{\eta}_{j-1,1}^{(0)} \times \boldsymbol{\eta}_{j}+\mathcal{O}\left(\Lambda_{X, Y}^{-3}\right),
$$

where $\boldsymbol{\eta}_{l, 1}^{(0)}=\sum_{j=1}^{l} \boldsymbol{\eta}_{j}$. The above expression can be proven by induction in $l$. Namely, we assume its validity for $l-1$ and compute $\boldsymbol{\eta}_{l, 1}$ from

$$
e^{-i \boldsymbol{\sigma} \cdot \boldsymbol{\eta}_{l, 1} / 2}=e^{-i \boldsymbol{\sigma} \cdot \boldsymbol{\eta}_{l} / 2} e^{-i \boldsymbol{\sigma} \cdot \boldsymbol{\eta}_{l-1,1} / 2},
$$

as in Eq. (E1). Equation (64) will follow from Eq. (E2) if the rotation angles are expressed in terms of displacements using Eqs. (55) and (63). The enclosed area is defined by

$$
A \mathbf{e}_{z}=\frac{1}{2} \sum_{j=2}^{l} \Delta \mathbf{r}_{j-1,1} \times \Delta \mathbf{r}_{j},
$$

where $\Delta \mathbf{r}_{j, 1}=\sum_{i=1}^{j} \Delta \mathbf{r}_{i}$ (Fig. 18).
*Electronic address: oleg.zaitsev@physik.uni-regensburg.de

${ }^{1}$ I. Žutić, J. Fabian, and S. Das Sarma, Rev. Mod. Phys. 76, 323 (2004), and references therein.

${ }^{2}$ E. I. Rashba, Fiz. Tverd. Tela (Leningrad) 2, 1224 (1960) [Sov. Phys. Solid State 2, 1109 (1960)].

${ }^{3}$ Y. A. Bychkov and E. I. Rashba, J. Phys. C 17, 6039 (1984).

${ }^{4}$ G. Dresselhaus, Phys. Rev. 100, 580 (1955).

${ }^{5}$ M. I. D’yakonov and V. I. Perel', Fiz. Tverd. Tela (Leningrad) 13, 3581 (1971) [Sov. Phys. Solid State 13, 3023 (1972)].

${ }^{6}$ M. I. D’yakonov and Y. Yu. Kachorovskii, Fiz. Tekh. Poluprovodn. (S.-Peterburg) 20, 178 (1986) [Sov. Phys. Semicond. 20, 110 (1986)].

${ }^{7}$ G. Lommer, F. Malcher, and U. Rössler, Phys. Rev. Lett. 60, 728 (1988).

${ }^{8}$ E. A. de Andrada e Silva, G. C. La Rocca, and F. Bassani, Phys. Rev. B 50, 8523 (1994).

${ }^{9}$ S. Datta and B. Das, Appl. Phys. Lett. 56, 665 (1990).

${ }^{10}$ F. Mireles and G. Kirczenow, Phys. Rev. B 64, 024426 (2001).

${ }^{11}$ J. Nitta, F. E. Meijer, and H. Takayanagi, Appl. Phys. Lett. 75, 695 (1999).

${ }^{12}$ D. Frustaglia and K. Richter, Phys. Rev. B 69, 235310 (2004).

${ }^{13}$ M. Governale, D. Boese, U. Zülicke, and C. Schroll, Phys. Rev. B 65, 140403(R) (2002).

${ }^{14}$ A. A. Kiselev and K. W. Kim, Appl. Phys. Lett. 78, 775 (2001).

${ }^{15}$ M. Governale, F. Taddei, and R. Fazio, Phys. Rev. B 68, 155324 (2003).

${ }^{16}$ P. Sharma and P. W. Brouwer, Phys. Rev. Lett. 91, 166801 (2003).

${ }^{17}$ J. Sinova, D. Culcer, Q. Niu, N. A. Sinitsyn, T. Jungwirth, and A. H. MacDonald, Phys. Rev. Lett. 92, 126603 (2004).
${ }^{18}$ S. Murakami, N. Nagaosa, and S.-C. Zhang, Science 301, 1348 (2003).

${ }^{19}$ V. N. Golovach, A. Khaetskii, and D. Loss, Phys. Rev. Lett. 93, 016601 (2004).

${ }^{20}$ J. Luo, H. Munekata, F. F. Fang, and P. J. Stiles, Phys. Rev. B 38, 10142 (1988).

${ }^{21}$ J. Nitta, T. Akazaki, H. Takayanagi, and T. Enoki, Phys. Rev. Lett. 78, 1335 (1997).

${ }^{22}$ G. Engels, J. Lange, T. Schäpers, and H. Lüth, Phys. Rev. B 55, R1958 (1997).

${ }^{23}$ T. Schäpers, J. Knobbe, and V. A. Guzenko, Phys. Rev. B 69, 235323 (2004).

${ }^{24}$ C. Schierholz, R. Kürsten, G. Meier, T. Matsuyama, and U. Merkt, Phys. Status Solidi B 233, 436 (2002).

${ }^{25}$ J. B. Miller, D. M. Zumbühl, C. M. Marcus, Y. B. Lyanda-Geller, D. Goldhaber-Gordon, K. Campman, and A. C. Gossard, Phys. Rev. Lett. 90, 076807 (2003).

${ }^{26}$ F. E. Meijer, A. F. Morpurgo, T. M. Klapwijk, T. Koga, and J. Nitta, Phys. Rev. B 70, 201307(R) (2004).

${ }^{27}$ G. M. Minkov, A. V. Germanenko, O. E. Rut, A. A. Sherstobitov, L. E. Golub, B. N. Zvonkov, and M. Willander, Phys. Rev. B 70, 155323 (2004).

${ }^{28}$ S. Hikami, A. I. Larkin, and Y. Nagaoka, Prog. Theor. Phys. 63, 707 (1980).

${ }^{29}$ G. Bergmann, Phys. Rep. 107, 1 (1984).

${ }^{30}$ S. Chakravarty and A. Schmid, Phys. Rep. 140, 193 (1986).

${ }^{31}$ B. Hackens, J. P. Minet, S. Faniel, G. Farhi, C. Gustin, J. P. Issi, J. P. Heremans, and V. Bayot, Phys. Rev. B 67, 121403(R) (2003).

${ }^{32}$ D. M. Zumbühl, J. B. Miller, C. M. Marcus, K. Campman, and A. 
C. Gossard, Phys. Rev. Lett. 89, 276803 (2002).

${ }^{33}$ O. Voskoboynikov, C. P. Lee, and O. Tretyak, Phys. Rev. B 63, 165306 (2001).

${ }^{34}$ M. Governale, Phys. Rev. Lett. 89, 206802 (2002).

${ }^{35}$ E. N. Bulgakov and A. F. Sadreev, Pis'ma Zh. Eksp. Teor. Fiz. 78, 911 (2003) [JETP Lett. 78, 443 (2003)].

${ }^{36}$ A. I. Saichev, H. Ishio, A. F. Sadreev, and K.-F. Berggren, J. Phys. A 35, L87 (2002).

${ }^{37}$ A. V. Khaetskii and Y. V. Nazarov, Phys. Rev. B 61, 12639 (2000).

${ }^{38}$ L. M. Woods, T. L. Reinecke, and Y. Lyanda-Geller, Phys. Rev. B 66, 161318(R) (2002).

${ }^{39}$ T. P. Pareek and P. Bruno, Phys. Rev. B 65, 241305(R) (2002); B. K. Nikolić and S. Souma, Phys. Rev. B 71, 195328 (2005).

${ }^{40}$ P. W. Brouwer, J. N. H. J. Cremers, and B. I. Halperin, Phys. Rev. B 65, 081302 (R) (2002).

${ }^{41}$ V. I. Fal'ko and T. Jungwirth, Phys. Rev. B 65, 081306(R) (2002).

${ }^{42}$ I. L. Aleiner and V. I. Fal'ko, Phys. Rev. Lett. 87, 256801 (2001).

${ }^{43}$ J.-H. Cremers, P. W. Brouwer, and V. I. Fal'ko, Phys. Rev. B 68, 125329 (2003).

${ }^{44}$ O. Zaitsev and K. Richter, Mater. Sci. 22, 469 (2004).

${ }^{45}$ O. Zaitsev, D. Frustaglia, and K. Richter, Phys. Rev. Lett. 94, 026809 (2005).

${ }^{46}$ C.-H. Chang, A. G. Mal'shukov, and K. A. Chao, Phys. Lett. A 326, 436 (2004).

${ }^{47}$ C.-H. Chang, A. G. Mal'shukov, and K. A. Chao, Phys. Rev. B 70, 245309 (2004).

${ }^{48}$ H. U. Baranger, R. A. Jalabert, and A. D. Stone, Phys. Rev. Lett. 70, 3876 (1993).

${ }^{49}$ H. U. Baranger, R. A. Jalabert, and A. D. Stone, Chaos 3, 665 (1993).

${ }^{50}$ K. Richter and M. Sieber, Phys. Rev. Lett. 89, 206801 (2002).

${ }^{51}$ R. G. Littlejohn and W. G. Flynn, Phys. Rev. A 45, 7697 (1992).

${ }^{52}$ H. Frisk and T. Guhr, Ann. Phys. (N.Y.) 221, 229 (1993).

${ }^{53}$ C. Amann and M. Brack, J. Phys. A 35, 6009 (2002).

${ }^{54}$ M. Pletyukhov, C. Amann, M. Mehta, and M. Brack, Phys. Rev. Lett. 89, 116601 (2002).

${ }^{55}$ O. Zaitsev, J. Phys. A 35, L721 (2002).

${ }^{56}$ M. Pletyukhov and O. Zaitsev, J. Phys. A 36, 5181 (2003).

${ }^{57}$ J. Cserti, A. Csordás, and U. Zülicke, Phys. Rev. B 70, 233307 (2004).

${ }^{58}$ M. V. Berry and K. E. Mount, Rep. Prog. Phys. 35, 315 (1972).

${ }^{59}$ J. R. Klauder, Phys. Rev. D 19, 2349 (1979).

${ }^{60}$ E. A. Kochetov, J. Math. Phys. 36, 4667 (1995).

${ }^{61}$ T. T. Wu and C. N. Yang, Phys. Rev. D 12, 3845 (1975).

${ }^{62}$ J. Bolte and S. Keppeler, Phys. Rev. Lett. 81, 1987 (1998).

${ }^{63}$ J. Bolte and S. Keppeler, Ann. Phys. (N.Y.) 274, 125 (1999).

${ }^{64}$ D. S. Fisher and P. A. Lee, Phys. Rev. B 23, R6851 (1981).

${ }^{65}$ M. Brack and R. K. Bhaduri, Semiclassical Physics, Vol. 96 of Frontiers in Physics (Westview Press, Boulder, Colorado, 2003).

${ }^{66}$ H. U. Baranger, D. P. DiVincenzo, R. A. Jalabert, and A. D. Stone, Phys. Rev. B 44, 10637 (1991).

${ }^{67}$ S. F. Nielsen, P. Dahlqvist, and P. Cvitanović, J. Phys. A 32, 6757 (1999).
${ }^{68}$ M. Sieber and K. Richter, Phys. Scr., T T90, 128 (2001).

${ }^{69}$ G. Bergmann, Solid State Commun. 42, 815 (1982).

${ }^{70}$ G. E. Pikus and A. N. Titkov, in Optical Orientation, edited by F. Meier and B. P. Zakharchenya (North-Holland, Amsterdam, 1984).

${ }^{71}$ S. Müller, Eur. Phys. J. B 34, 305 (2003).

${ }^{72}$ V. S. Varadarajan, Lie Groups, Lie Algebras and their Representations (Prentice-Hall, Englewood Cliffs, NJ, 1974).

${ }^{73}$ R. M. Wilcox, J. Math. Phys. 8, 962 (1967).

${ }^{74}$ D. Frustaglia, M. Hentschel, and K. Richter, Phys. Rev. B 69, 155327 (2004)

${ }^{75}$ I. S. Gradshteyn and I. M. Ryzhik, Tables of Integrals, Series, and Products (Academic, New York, 1965).

${ }^{76}$ R. P. Mondescu and M. Muthukumar, Phys. Rev. E 57, 4411 (1998).

${ }^{77}$ M. Turek and K. Richter, J. Phys. A 36, L455 (2003).

${ }^{78}$ N. S. Averkiev and L. E. Golub, Phys. Rev. B 60, 15582 (1999).

${ }^{79}$ F. G. Pikus and G. E. Pikus, Phys. Rev. B 51, 16928 (1995).

${ }^{80}$ J. Schliemann, J. C. Egues, and D. Loss, Phys. Rev. Lett. 90, 146801 (2004).

${ }^{81}$ Another derivation involving a slightly different approach can be found in Ref. 30.

${ }^{82} \mathbf{C}_{[\mathbf{q}, \mathbf{p}]}(t)$ is measured in units of frequency.

${ }^{83}$ Equation (19) is only valid if there are no families of direct trajectories (without reflections) connecting the leads (Ref. 49).

${ }^{84}$ In Ref. 50 the diagonal contribution is defined differently and includes the classical part.

${ }^{85} \mathrm{~A}$ generalized phase-space approach for the loop contributions, also valid for hyperbolic systems with smooth potential, is considered in Ref. 77.

${ }^{86}$ This means that the orbital Hamiltonian $H^{0}$ has time-reversal symmetry and that $\mathbf{C}(\mathbf{q}, \mathbf{p})$ is an odd function of the velocity components, i.e., it changes sign upon time reversal.

${ }^{87}$ The cyclotron radius must be larger than the system size.

${ }^{88}$ Semiclassical description of diffusive systems implies that the Fermi wavelength is much smaller than the mean free path.

${ }^{89}$ It was argued in Ref. 47 that the saturation is due to the nearly periodic orbits, which have a finite measure in integrable systems.

${ }^{90}$ The interplay between the Rashba and the Dresselhaus terms in extended diffusive systems was studied in Ref. 78 (spin relaxation) and in Ref. 79 (weak antilocalization).

${ }^{91}$ In this this case the system possesses an additional conserved quantity (Ref. 80).

${ }^{92}$ The partial coefficients $\left|t_{n m}\right|^{2}$ and $\left|r_{n m}\right|^{2}$ are invariant, as well.

${ }^{93}$ The requirement that the incoming and outgoing channels be the same was neglected.

${ }^{94}$ The Zeeman contribution due to spin coupling to an external (possibly inhomogeneous) magnetic field appears only in the on-site terms since it does not involve spacial derivatives. On the contrary, the SO coupling depending on the momentum $\mathbf{p}$ contributes mainly to the hopping terms.

${ }^{95}$ It took minutes to produce the semiclassical curves in Figs. 16 and 17 , and a couple of weeks for their quantum counterparts. 